\title{
Simplified models of dark matter with a long-lived co-annihilation partner
}

\author{
Valentin V. Khoze, ${ }^{a}$ Alexis D. Plascencia ${ }^{a}$ and Kazuki Sakurai ${ }^{a, b}$ \\ ${ }^{a}$ Institute for Particle Physics Phenomenology, Department of Physics, Durham University, \\ South Road, Durham DH1 3LE, U.K. \\ ${ }^{b}$ Institute of Theoretical Physics, Faculty of Physics, University of Warsaw, \\ ul. Pasteura 5, PL-02-093 Warsaw, Poland \\ E-mail: valya.khoze@durham.ac.uk, \\ a.d.plascencia-contreras@durham.ac.uk, kazuki.sakurai@fuw.edu.pl
}

ABSTRACT: We introduce a new set of simplified models to address the effects of 3-point interactions between the dark matter particle, its dark co-annihilation partner, and the Standard Model degree of freedom, which we take to be the tau lepton. The contributions from dark matter co-annihilation channels are highly relevant for a determination of the correct relic abundance. We investigate these effects as well as the discovery potential for dark matter co-annihilation partners at the LHC. A small mass splitting between the dark matter and its partner is preferred by the co-annihilation mechanism and suggests that the co-annihilation partners may be long-lived (stable or meta-stable) at collider scales. It is argued that such long-lived electrically charged particles can be looked for at the LHC in searches of anomalous charged tracks. This approach and the underlying models provide an alternative/complementarity to the mono-jet and multi-jet based dark matter searches widely used in the context of simplified models with $s$-channel mediators. We consider four types of simplified models with different particle spins and coupling structures. Some of these models are manifestly gauge invariant and renormalizable, others would ultimately require a UV completion. These can be realised in terms of supersymmetric models in the neutralino-stau co-annihilation regime, as well as models with extra dimensions or composite models.

KEywords: Beyond Standard Model, Cosmology of Theories beyond the SM

ArXiv EPRINT: 1702.00750 


\section{Contents}

1 Introduction 1

2 Simplified models $\quad 3$

3 Co-annihilation $\quad 5$

4 Experimental signatures $\quad 7$

$\begin{array}{lll}4.1 & \text { Direct detection } & 7\end{array}$

$\begin{array}{lll}4.2 & \text { Indirect detection } & 7\end{array}$

4.3 Collider searches 8

5 Results $\quad 11$

5.1 Model 1a: Majorana fermion dark matter 11

5.2 Model 1b: effect of L-R mixing 13

$\begin{array}{ll}5.3 \text { Model 2: scalar dark matter } & 15\end{array}$

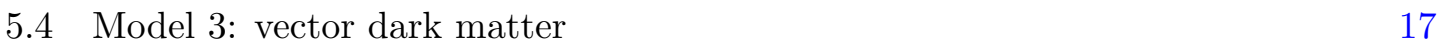

$\begin{array}{llr}6 & \text { Conclusions } & 18\end{array}$

$\begin{array}{ll}\text { A Indirect detection limits for Model } 3 & 19\end{array}$

B Limits in the mass vs lifetime plane $\quad 20$

\section{Introduction}

The existence of dark matter (DM) in the Universe has been established by a number of astrophysical observations based on gravitational interactions. Using the standard model of cosmology, the data collected by the Planck mission [1] implies that DM constitutes nearly $85 \%$ of the total matter content in the universe. Nevertheless, a microscopic description of DM by a fundamental particle theory is still missing and the nature of dark matter remains largely unknown. There is a well-established approach to search for dark matter which relies on the three distinct detection strategies: the direct detection, the indirect detection and DM searches at colliders. ${ }^{1}$ The direct detection searches use underground experiments that measure nucleon recoil in order to detect the interaction between DM and nucleons. The indirect detection strategy uses experiments that look for an astrophysical signal coming from decays or annihilation of DM particles into the Standard Model (SM) particles. Finally, dark matter is actively searched at colliders, presently at the LHC, with the aim to produce DM particles in proton collisions. As the SM does not contain a

\footnotetext{
${ }^{1}$ For a classic review of particle DM candidates and the experimental search strategies see e.g. [2].
} 
viable DM candidate, any evidence of DM production at colliders would be a signal of new physics, the discovery of which is arguably one of the most important goals in the field.

Despite an intense experimental effort and surveys of these three directions, the dark matter has so far proven to be elusive. The no-observation of DM is starting to put some pressure on the so-called WIMP Miracle paradigm, which posits that the observed relic abundance can be explained by DM candidates which are weakly interacting massive particles (WIMPs) with masses in the $10 \mathrm{~s}$ of $\mathrm{GeV}$ to a few $\mathrm{TeV}$ range (assuming simple $2 \rightarrow 2 \mathrm{DM}$ annihilation to SM particles and the standard thermal freeze-out mechanism). A growing number of such WIMP models of DM are being strongly constrained by, or at least show tension with the experimental limits, including supersymmetric DM realisations discussed in [3-14] as well as other models considered in e.g. [15, 16].

Our ignorance of the dark sector structure and the negative experimental results for DM searches have motivated more model-independent studies which fall into two categories. The first is based on exploiting effective operators describing the low energy interactions between the DM and the SM particles [17-30]. This EFT approach manifestly does not depend on the UV structure of the (unknown) microscopic dark sector theory and works well when applied to the low energy experiments, such as the direct detection. However, the EFT approximation often breaks down when studying collider signatures since the cutoff of the effective field theory may not be larger than the LHC's energy scale or the dark sector often requires a new mediator particle other than the DM which may dramatically alter the collider signature itself [31-33].

The alternative framework is the simplified model approach, in which sets of phenomenological models are constructed with a minimal particle content to describe various experimental signatures. This approach turns out to be very useful and searches for dark matter at colliders are now commonly described in terms of simplified models with scalar, pseudo-scalar, vector and axial-vector mediators [34-37]. These simplified models have become the main vehicle for interpreting DM searches at the LHC $[38,39]$ and for projecting the DM reach of future hadron colliders [40-42].

These simplified models can be viewed as arising from integrating out the irrelevant particles and taking a certain limit of the more detailed microscopic theories. The dependence on specific details of any particular UV embedding in this case is by definition beyond the scope of the simplified models settings. An interesting question to ask is of course whether and which types of UV completions of specific simplified models are possible and if the additional degrees of freedom would affect the simplified model predictions at particular collider scales. For recent examples and studies of such 'next-to-simplified models' we refer the reader to refs. [43-49].

The simplified models used by the LHC experiments and aggregated by the ATLASCMS DM Forum and the LHC DM Working Group [38, 39] are conventionally classified based on the type of mediator particles that connect the DM to the SM particles. However, this classification may miss an effect of co-annihilation that can be important to determine the DM relic density [50]. In the scenario where the co-annihilation is operative, a charged (or coloured) particle is introduced in addition to the DM, which we call the co-annihilation partner. Since the interaction between the co-annihilation partner and the SM particles is 
unsuppressed, they annihilate efficiently into the SM particles in the early universe. Due to the thermal transition between the DM and the co-annihilation partner, the DM density is also reduced. This scenario does not require conventional interactions between the DM and the ordinary particles through a mediator, and otherwise severe experimental constraints, can easily be avoided. Simplified model studies addressing DM co-annihilation and collider signatures so far have mostly focused on the coloured co-annihilation partners [50-56], with only few exceptions as in [57] (or in [58] including semi-annihilation effects between two different components of dark matter, e.g. Vector Vector $\rightarrow$ Vector Scalar).

The collider signature is also different in the co-annihilation scenario from the usual DM simplified models. Since the co-annihilation partner couples to the SM sector with an unsuppressed coupling, the production rate is much higher for the co-annihilation partners than for DM particles. Moreover, the co-annihilation partner can be long-lived at colliders because its mass difference from the DM mass is small and the decay rate incurs a significant phase space suppression. This may be the case in particular when the co-annihilation partner has a contact interaction with the DM particle and a $\tau$-lepton, since if the mass difference is smaller than $m_{\tau}$, the co-annihilation partner decays into multi-body final states via an off-shell $\tau$, leading to a strong phase space suppression. This situation is familiar in supersymmetric (SUSY) theories with the stau co-annihilation [59-65].

In this paper, we introduce a class of simplified models that enables us to study the phenomenology of the dark sector containing a co-annihilation partner. Inspired in part by the neutralino-stau co-annihilation mechanism in SUSY theories, we want to recreate it in more general settings using a new class of simplified model. In section 2 we will define four types of simplified models with different particle spins and coupling structures and assume the existence of a contact interaction involving the DM particle, its co-annihilation partner and the SM $\tau$-lepton. Our simplified model choices include a fermionic DM with a scalar co-annihilation partner, a scalar DM with a fermionic co-annihilation partner and a vector DM with a fermionic co-annihilation partner. Some of these models are manifestly gauge invariant and renormalizable, others are supposed to descend from a more detailed UV complete theory with or without supersymmetry, some may be realised as a certain limit of composite models, or descent from models with large extra dimensions. The expressions for our Simplified Model Lagrangians and the definitions of the free parameters characterising the models can be found in eqs. (5.1), (5.4)-(5.7) in section 5. The section 3 explains the coannihilation mechanism for computing the DM relic density in the context of our simplified models. This is followed by a general overview of experimental signatures for direct and indirect detection and collider searches in section 4. Our main results are presented and discussed in section 5. Finally in section 6 we draw our conclusions.

\section{Simplified models}

To implement the Dark Matter co-annihilation mechanism we consider dark sectors which include two distinct degrees of freedom: the DM particle, $\chi$, and the charged co-annihilation partner (CAP), $\eta^{( \pm)}$. We assume that both of these dark sector particles have odd parity under a $Z_{2}$ symmetry to ensure the stability of the dark matter $\chi$. Our simplified models 


\begin{tabular}{|c|c|c|c|}
\hline \multicolumn{4}{|c|}{ Model-1a } \\
\cline { 1 - 2 } Component & Field & Charge & Interaction (5.1) \\
\hline DM & Majorana fermion $(\chi)$ & $Y=0$ & \multirow{2}{*}{$\phi^{*}\left(\chi \tau_{R}\right)+$ h.c. } \\
\hline CAP & Complex scalar $(\phi)$ & $Y=-1$ & \\
\hline
\end{tabular}

\begin{tabular}{|c|c|c|c|}
\hline \multicolumn{4}{|c|}{ Model-1b } \\
\hline Component & Field & Charge & Interaction $(5.4)-(5.5)$ \\
\hline DM & Majorana fermion $(\chi)$ & $Q=0$ & \multirow{2}{*}{$\phi^{*}\left(\chi \tau_{R}\right)+\phi^{*}\left(\chi \tau_{L}\right)+$ h.c. } \\
\hline CAP & Complex scalar $(\phi)$ & $Q=-1$ & \\
\hline
\end{tabular}

\begin{tabular}{|c|c|c|c|}
\hline \multicolumn{4}{|c|}{ Model-2 } \\
\cline { 1 - 2 } Component & Field & Charge & Interaction (5.6) \\
\hline DM & Real scalar $(S)$ & $Y=0$ & \multirow{2}{*}{$S\left(\bar{\Psi} P_{R} \tau\right)+$ h.c. } \\
\hline CAP & Dirac fermion $(\Psi)$ & $Y=-1$ & \\
\hline
\end{tabular}

\begin{tabular}{|c|c|c|c|}
\hline \multicolumn{4}{|c|}{ Model-3 } \\
\cline { 1 - 2 } Component & Field & Charge & Interaction (5.7) \\
\hline DM & Vector $\left(V_{\mu}\right)$ & $Y=0$ & \multirow{2}{*}{$V_{\mu}\left(\bar{\Psi} \gamma^{\mu} P_{R} \tau\right)+$ h.c. } \\
\hline CAP & Dirac fermion $(\Psi)$ & $Y=-1$ & \\
\hline
\end{tabular}

Table 1. Simplified Models of DM with a colourless co-annihilation partner (CAP).

are defined by the three-point interactions between $\chi, \eta$ and the $\tau$-lepton of the Standard Model sector,

$$
\mathcal{L} \supset g_{\mathrm{DM}} \chi \eta \tau+\text { h.c. . }
$$

Here $g_{\mathrm{DM}}$ denotes the dark sector coupling constant which we take to be real and we also note that $\eta$ has a non-vanishing $\tau$-lepton number. Restricting the particle content of our simplified models to spins not higher than 1 , we consider three possible spin assignments $^{2}$ for the $(\chi, \eta)$ pair: $\left(\frac{1}{2}, 0\right),\left(0, \frac{1}{2}\right)$ and $\left(1, \frac{1}{2}\right)$. The corresponding simplified DM-co-annihilation models we wish to consider are summarised in table 1.

A note on notation: we use $\chi$ to denote the DM particle and $\eta$ (or $\eta^{ \pm}$) for the coannihilation particle in general. For the simplified models in table 1 we have $\chi=\left\{\chi, S, V_{\mu}\right\}$ and $\eta=\{\phi, \Psi\}$ depending on the choice of the model.

For the $\left(\frac{1}{2}, 0\right)$ spin assignment we consider the case where the dark matter is a Majorana fermion, $\chi$, and the co-annihilation partner is a complex scalar field, $\phi$, bearing in mind the similarity of this case with the neutralino-stau co-annihilation picture in SUSY models,

\footnotetext{
${ }^{2}$ An additional potential assignment $\left(\frac{1}{2}, 1\right)$ leads to $\eta$ being an electrically charged vector boson which prevent us from finding an $\mathrm{SU}(2)_{\mathrm{L}} \times \mathrm{U}(1)_{\mathrm{Y}}$ invariant operator for eq. (2.1). We therefore will not consider this option further.
} 
where $\chi$ plays the role of the lightest neutralino, and the scalar $\phi$ is the stau. In the simplest realisation of this simplified model, which we refer to as the Model-1a in table 1, the Yukawa interactions (2.1) between the dark sector particles $\chi, \phi$ and the SM involve only the right-handed component of the $\tau$-lepton, $\tau_{R}$, hence the co-annihilation scalar $\phi$ is an $\mathrm{SU}(2)_{\mathrm{L}}$-singlet. At the same time, the second realisation - the Model-1b - involves interactions with both left- and right-handed $\tau$-leptons, and hence the stau-like scalar dark partner $\phi$ is charged under the $\mathrm{SU}(2)_{\mathrm{L}}$. The Simplified Model-1a is a UV-consistent theory as it stands; on the other hand, the Model-1b should ultimately be embedded into a more fundamental microscopic theory in the UV to be consistent with the gauge invariance under $\mathrm{SU}(2)_{\mathrm{L}}$. One such embedding can for example be a supersymmetric model with an operational neutralino-stau co-annihilation mechanism.

The simplified model corresponding to the $\left(0, \frac{1}{2}\right)$ spin assignment is called Model-2, in which we introduce a real scalar $S$ as the dark matter and a Dirac fermion, $\Psi$, as the co-annihilation partner, assuming they couple together with $\tau_{R}$. Model-3 is constructed for the $\left(1, \frac{1}{2}\right)$ spin assignment that introduces a real vector, $V_{\mu}$, for the dark matter and a Dirac fermion, $\Psi$, for the co-annihilation partner, assuming again the interaction with $\tau_{R}$. These two simplified models can be realised in models of extra dimensions and/or composite models as we will outline in section 5 .

The simplified models 1a, 2 and 3 constructed above have the following free parameters: the dark matter mass, $m_{\mathrm{DM}} \equiv m_{\chi}$, the mass splitting, $\Delta M=M_{\eta}-m_{\chi}$, and the dark sector coupling, $g_{\mathrm{DM}}$. In Model-1b we fix the dark sector coupling to be the $\mathrm{U}(1)_{\mathrm{Y}}$ gauge coupling $\left(g_{\mathrm{DM}}=g^{\prime}\right)$. Instead, we introduce the L-R mixing angle, $\theta$, which controls the relative strength of the coupling to $\tau_{L}$ and $\tau_{R}$, as we will discuss later in more detail. The simplified model Lagrangians and the parameter definitions are given in eq. (5.1) for Model 1a, eqs. (5.4)-(5.5) for Model 1b, eq. (5.6) for Model 2 and in (5.7) for Model 3.

\section{Co-annihilation}

The effect of co-annihilation can be understood qualitatively in the space of simplified model parameters. First of all, it is worth noting that $\chi$ couples to the SM sector only through the operator eq. (2.1), whereas $\eta^{ \pm}$interacts with the SM particles also via the electromagnetic and weak gauge interactions. In our simplified models, there is a unique channel for the DM pair annihilation: $\chi \chi \rightarrow \tau^{+} \tau^{-}$, as shown in the left diagram in figure 1 . For small $g_{\mathrm{DM}}$, the DM pair annihilation is highly suppressed because the rate of this process is proportional to $g_{\mathrm{DM}}^{4}$. For our simplified models 1a,b and 2 where the dark matter is a Majorana fermion or a real scalar $(\chi=\{\chi, S\})$, there is another suppression factor. The initial state in both these cases forms a spin-0 state (due to the Pauli blocking in the Majorana case). To conserve the angular momentum, the $\tau^{+} \tau^{-}$pair in the final state must have the opposite chiralities in the $s$-wave contribution, hence meaning that this contribution is suppressed by $m_{\tau}^{2}$ (chiral suppression). The dominant contribution then comes from the $p$-wave for a Majorana DM and $d$-wave for a scalar DM, which are suppressed by the factor $v^{2}$ and $v^{4}$, respectively, where $v$ is the average of the relative velocity of the annihilating DM particles. 

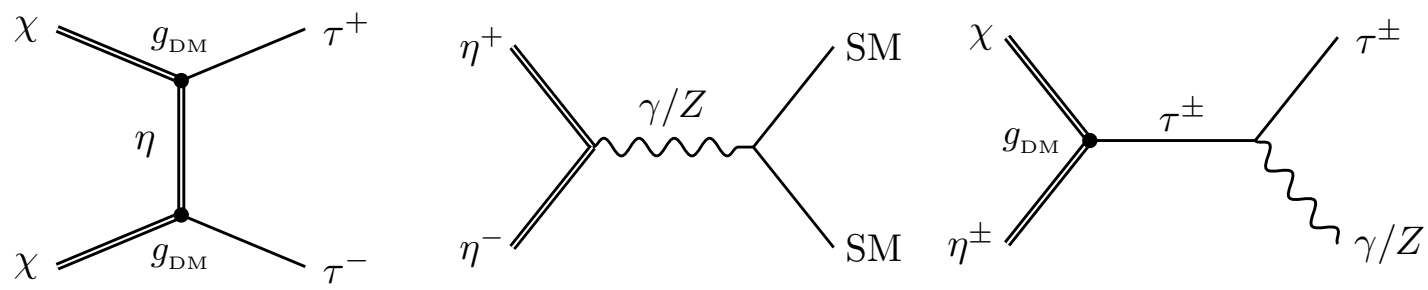

Figure 1. Annihilation and co-annihilation processes.

Unlike the DM pair annihilation, the annihilation of the CAP particles, $\eta \eta \rightarrow S M$ particles, proceeds via the electromagnetic or weak gauge interactions, as indicated in the second diagram of figure 1 . As such, the $\eta \eta$ annihilation can have much larger rates than the first process in figure 1 at small $g_{\mathrm{DM}}$. For a small but non-vanishing values of $g_{\mathrm{DM}}$, there are transition processes between $\eta$ and $\chi: \eta+\mathrm{SM} \leftrightarrow \chi+\mathrm{SM}$. These processes are in general much more efficient than annihilation processes, since the number density of light SM particles is not Boltzmann suppressed at the time of freeze-out. As long as the mass splitting, $\Delta M$, is small, the transition process effectively equalises the number densities of $\chi$ and $\eta$, and the DM density (in the unit of the entropy density) freezes out when the annihilation of $\eta$ is decoupled. We therefore find that in the region of small $g_{\mathrm{DM}}$, the DM relic density is not sensitive to $g_{\mathrm{DM}}$ and determined mainly by $\Delta M$ and $\sigma(\eta \eta \rightarrow S M$ particles $) \times v$.

As $g_{\mathrm{DM}}$ approaches the $\mathrm{U}(1)_{\mathrm{Y}}$ gauge coupling, $g^{\prime}$, the co-annihilation process $\chi \eta \rightarrow$ $S M$ particles becomes important (see, for example, the right diagram in figure 1). The rate of this process is proportional to $g_{\mathrm{DM}}^{2}$. As in the previous process, this process is only effective when $\Delta M$ is small as we will see below more explicitly.

For even higher values of $g_{\mathrm{DM}}$, the dark matter pair annihilation, $\chi \chi \rightarrow \tau^{+} \tau^{-}$, can become important, since the annihilation rate is proportional to $g_{\mathrm{DM}}^{4}$. However, as we have discussed above, for $\chi=\{\chi, S\}$, this process can never become very large because it is velocity suppressed. However it can be dominant for the vector DM case $\chi=V_{\mu}$. Unlike the other channels, the contribution of this process is independent of $\Delta M$.

As it is well known, the DM relic abundance scales as

$$
\Omega_{\mathrm{DM}} h^{2} \propto\left\langle\sigma_{\text {eff }} v\right\rangle^{-1},
$$

where $\left\langle\sigma_{\text {eff }} v\right\rangle$ is the thermal average of the effective annihilation cross-section that is given by $[66]$

$$
\begin{gathered}
\sigma_{\text {eff }} v=\frac{1}{\left(\mathrm{~g}_{\chi}+\overline{\mathrm{g}}_{\eta}\right)^{2}}\left[\mathrm{~g}_{\chi}^{2} \cdot \sigma\left(\chi \chi \rightarrow \tau^{+} \tau^{-}\right)+\mathrm{g}_{\chi} \overline{\mathrm{g}}_{\eta} \cdot \sigma(\chi \eta \rightarrow S M \text { particles })+\right. \\
\left.+\overline{\mathrm{g}}_{\eta}^{2} \cdot \sigma(\eta \eta \rightarrow S M \text { particles })\right] v
\end{gathered}
$$

with

$$
\overline{\mathrm{g}}_{\eta}=\mathrm{g}_{\eta}\left(\frac{M_{\eta}}{m_{\chi}}\right)^{3 / 2} \exp \left(-\frac{\Delta M}{T}\right)
$$


where $\mathrm{g}_{\chi}$ and $\mathrm{g}_{\eta}$ denote the degrees of freedom of the fields $\chi$ and $\eta$, respectively, and should not be confused with the dark sector coupling $g_{\mathrm{DM}}$. Their explicit values are given as $\left(\mathrm{g}_{S}, \mathrm{~g}_{\chi}, \mathrm{g}_{\phi}, \mathrm{g}_{V_{\mu}}, \mathrm{g}_{\Psi}\right)=(1,2,2,3,4)$. Each line of eq. (3.2) corresponds to the different contribution discussed above and depicted in figure 1. The dependence of these contributions on $\Delta M$ can be found through $\overline{\mathrm{g}}_{\eta}$. Since the freeze-out occurs around $T \sim m_{\mathrm{DM}} / 25$, $\Delta M \lesssim m_{\mathrm{DM}} / 25$ is required in order not to have large suppressions for the processes $\chi \eta \rightarrow S M$ particles and $\eta \eta \rightarrow S M$ particles. In this study we are interested in the regime where the co-annihilation is operative, and we demand $\Delta M$ to be small. In our numerical study we compute $\Omega_{\mathrm{DM}} h^{2}$ using MicrOMEGAs 4.1 .5 [67] implementing the simplified models with help of FeynRules 2.0 [68] and LanHEP 3.2 [69].

\section{Experimental signatures}

\subsection{Direct detection}

Since the DM couples to the SM sector only through the interaction term eq. (2.1), the strength of experimental signatures is rather weak in general for the simplified models introduced in section 2. Direct detection experiments measure the nuclei recoil resulting from their interaction with dark matter, but such interactions involving DM with quarks and gluons are absent at tree-level in our simplified models. At one-loop level, the relevant operators may be generated. The Higgs mediating contributions are too small because the amplitude is suppressed by the product of the tau Yukawa coupling and the Yukawa coupling in the hadron sector. The relevant operators describing the interactions between the DM and the neutral gauge bosons are generated at dimension 6 at the lowest and suppressed by $1 / M_{\eta}^{2}$. For example, for the Majorana DM case, such an operator is given by the anapole moment operator $\mathcal{A} \bar{\chi} \gamma_{\mu} \gamma_{5} \chi \partial^{\nu} F_{\mu \nu}$. For $m_{\mathrm{DM}} \simeq 500 \mathrm{GeV}$ and $\Delta M / m_{\tau}<1$, the anapole moment is roughly given by $\mathcal{A} / g_{\mathrm{DM}}^{2} \sim 8 \cdot 10^{-7}\left[\mu_{N} \cdot \mathrm{fm}\right]$ [70], which is more than one order of magnitude smaller than the current limit obtained by LUX [71] and also smaller than the projected sensitivity of LZ [72], even for $g_{\mathrm{DM}}^{2}=1 .^{3}$ Although a dedicated study may shed some light on the future direct detection prospects for our simplified models, we shall postpone such a study to a future work.

\subsection{Indirect detection}

Indirect detection experiments are looking for high energy cosmic rays or neutrinos originated from the DM pair annihilation (or decay) in the present Universe. For the $2 \rightarrow 2$ topology, the only relevant process is $\chi \chi \rightarrow \tau^{+} \tau^{-}$shown by the right diagram of figure 1 . As mentioned in the previous section, for $\chi=\{\chi, S\}$ this process suffers from the chiral suppression, and the signal rate for the indirect detection goes below the experimental sensitivity. The chiral suppression is absent for $\chi=V_{\mu}$ (Model-3). In appendix A we compare the annihilation rate of $V_{\mu} V_{\mu} \rightarrow \tau^{+} \tau^{-}$with the current limit obtained by Fermi-LAT [76],

\footnotetext{
${ }^{3}$ The limits mentioned here assume the observed energy density of the DM. On the other hand, for $m_{\mathrm{DM}} \simeq 500 \mathrm{GeV}$ and $g_{\mathrm{DM}} \simeq 1$, all of our simplified models underproduce the $\chi$ particles. The actual constraints would therefore be even milder if this effect is taken into account.
} 


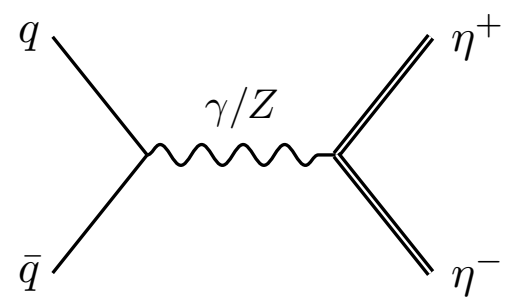

Figure 2. Co-annihilation partner (CAP) pair-production process.

taking into account the rescaling of the flux factor by the predicted relic abundance. We find that the annihilation rate in Model-3 is two orders of magnitude smaller than the current limit across the parameter region.

The $2 \rightarrow 3$ scattering, $\chi \chi \rightarrow \tau^{+} \tau^{-} \gamma$, may be more interesting in a small $\Delta M$ region. In this regime, the reaction rate of this process is enhanced in the following way. One of the DM particles can be converted into a slightly off-shell $\eta$ radiating off a soft tau, $\chi \rightarrow \eta^{ \pm} \tau^{\mp}$. This $\eta^{ \pm}$can then co-annihilate with the other $\chi$ particle via $\chi \eta^{ \pm} \rightarrow \tau^{ \pm} \gamma$ (see, for example, the third diagram in figure 1). Since the converted $\eta^{ \pm}$is only slightly off-shell, the propagator of $\eta^{ \pm}$is enhanced, and the energy distribution of the produced $\gamma$ has a peak around $m_{\mathrm{DM}} / 2$, which can be seen as a bump in a smoothly falling background. Although this signature is in principle promising, it has been shown that for $\Delta M \ll m_{\mathrm{DM}}$ the annihilation rate is nevertheless below the experimental sensitivities [70, 73-75]. For example, for the Majorana (scalar) DM with $m_{\mathrm{DM}}=500 \mathrm{GeV}$ and $\Delta M / m_{\tau}<1$, the annihilation rate is roughly given by $\left\langle v \sigma\left(\chi \chi \rightarrow \tau^{+} \tau^{-} \gamma\right)\right\rangle / g_{\mathrm{DM}}^{2} \sim 5 \cdot 10^{-29}\left(5 \cdot 10^{-28}\right)\left[\mathrm{cm}^{3} / \mathrm{s}\right]$, which is smaller than the current limits obtained by Fermi-LAT [76] and HESS [77], and also below the future sensitivity of CTA $[78,79]$ even for $g_{\mathrm{DM}}=1$ and assuming $\Omega_{\chi} h^{2}=$ $\Omega_{\mathrm{DM}} h^{2} \simeq 0.1197$. As in the direct detection case, we reserve the dedicated study on the prospects of the indirect detection sensitivity to our simplified models for a future work.

\subsection{Collider searches}

In general, DM particles can be produced in proton-proton collisions at the LHC and the experimental collaborations are looking for signatures of such DM production, usually involving mono- and multi-jets plus missing energy, or alternatively constraining a direct mediator production which could decay back into SM. In our simplified models of DM with colourless co-annihilation partners, however, no direct DM production processes are possible at tree level since the DM couples to the SM sector only via the interactions (2.1).

Unlike the DM particle, the co-annihilation $\eta$ particle couples to the SM sector via electro-weak gauge interactions, and $\eta$ can be pair-produced by exchanging off-shell neutral gauge bosons $q \bar{q} \rightarrow(\gamma / Z)^{*} \rightarrow \eta \eta$ as depicted in figure 2 . The production rate is independent of $g_{\mathrm{DM}}$ and is well-defined once the mass and quantum numbers of $\eta$ are specified. For our simplified models of DM with co-annihilation partners $\eta$, the latter are either a complex scalar or Dirac fermions. The $\eta$ production cross-sections $p p \rightarrow \eta \eta$ at the $8 \mathrm{TeV}$ and $13 \mathrm{TeV}$ LHC computed at leading order by MadGraph 5 [80] for our range of simplified models are 


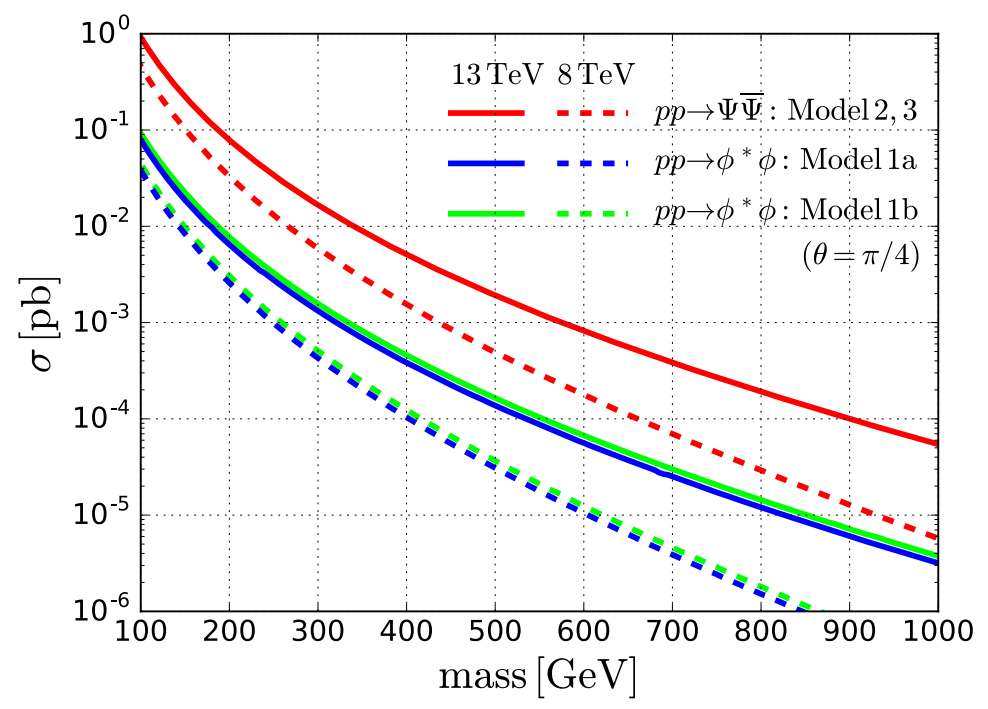

Figure 3. Collider cross-section $\sigma^{\mathrm{LO}}\left(p p \rightarrow \eta^{+} \eta^{-}\right)$for the simplified models defined in table 1 .

plotted in figure 3 as the function of the co-annihilation partner mass. It can be seen that the production cross-section in the fermion case is one order of magnitude higher than in the scalar case. This is because the scalar production suffers from velocity suppression near the threshold; we will further comment on this effect in section 5.3.

In the region where the co-annihilation is operative, $\Delta M$ is small and the decay products of $\eta$ will be too soft to be reconstructed. ${ }^{4}$ The standard strategy to trigger such events is to demand additional hard jet originated from the initial state QCD radiation. This leads to a distinct mono-jet plus large missing energy signature and the signal can (in favourable settings) be separated from the background. It is known that the mono-jet channel is powerful if $\eta$ has a colour charge, but for our colour-neutral $\eta$ this prospect is, as one would expect, quite pessimistic. For example, the study presented in [82] did not find any limit on the stau mass in the stau co-annihilation region in SUSY models using a mono-jet channel even for a $100 \mathrm{TeV} p p$ collider with a $3 \mathrm{ab}^{-1}$ integrated luminosity. In this paper we focus on the sensitivity at the LHC and aim to look for an alternative search channel.

As we have seen in section 3 , the effective co-annihilation mechanism in the dark sector imposes an upper bound on the mass splitting between the DM and the CAP particles, $\Delta M \lesssim m_{\mathrm{DM}} / 25$. Furthermore, if $\Delta M$ becomes smaller than the $\tau$-lepton mass, $m_{\tau}=1.777 \mathrm{GeV}$, the on-shell 2-body decay, $\eta^{ \pm} \rightarrow \chi \tau^{ \pm}$, is kinematically forbidden and the 3- and 4-body decay modes, $\eta^{ \pm} \rightarrow \chi \nu_{\tau} \pi^{ \pm}$and $\eta^{ \pm} \rightarrow \chi \nu_{\tau} \ell^{ \pm} \nu_{\ell}(\ell=e, \mu)$ shown in figure 4, become dominant. Since these 3 - and 4-body decays are suppressed by the off-shell intermediate propagators and the multi-body phase space, the $\eta$ decay rate becomes minuscule.

We show in figure 5 the lifetimes of $\eta^{ \pm}$computed with CalcHEP [83] as functions of $\Delta M$ for our simplified models of DM with a co-annihilation partner. As can be seen, the lifetimes

\footnotetext{
${ }^{4}$ The LHC phenomenology of a similar model in the opposite limit $\left(\Delta M \sim m_{\mathrm{DM}}\right)$ have been studied in [81].
} 

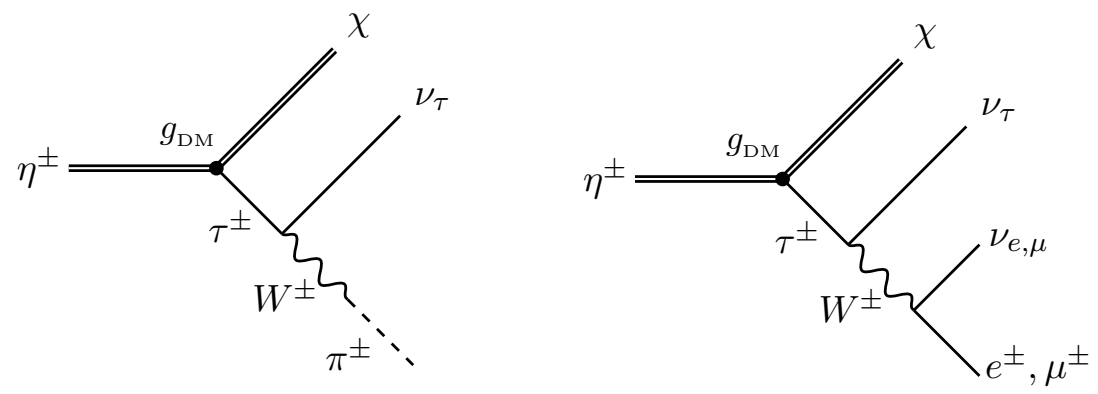

Figure 4. The 3-body and 4-body $\eta$-decays via an off-shell $\tau$ (and $W$ ).

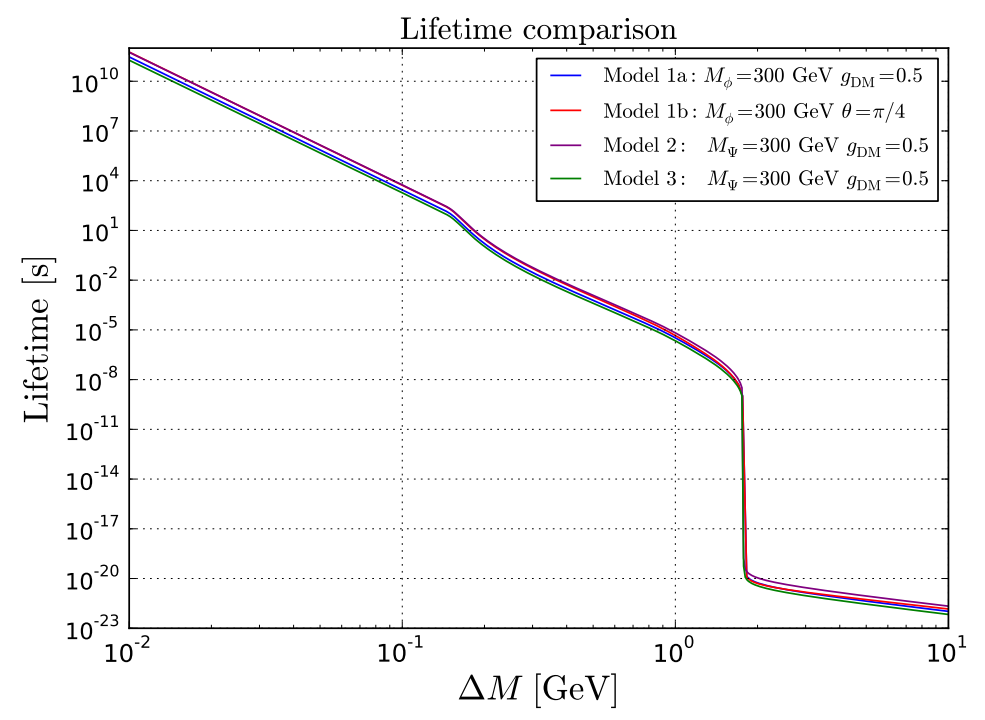

Figure 5. The lifetime of the co-annihilation partner $\eta^{ \pm}$as a function of the mass splitting $\Delta M=$ $M_{\eta}-m_{\chi}$. Model 1a (blue): $M_{\phi}=300 \mathrm{GeV}, g_{\mathrm{DM}}=0.5$, Model $1 \mathrm{~b}(\mathrm{red}): M_{\phi}=300 \mathrm{GeV}, \theta=\pi / 4$, Model 2 (purple): $M_{\Psi}=300 \mathrm{GeV}, g_{\mathrm{DM}}=0.5$, Model 3 (green): $M_{\Psi}=300 \mathrm{GeV}, g_{\mathrm{DM}}=0.5$.

quickly increase once $\Delta M$ crosses $m_{\tau}$ from above and reach $\sim 1 \mu s$ around $\Delta M \sim 1 \mathrm{GeV}$, for all simplified models. If the lifetime is of the order of $\mu s, \eta$ can reach the tracker and may leave anomalously highly ionizing tracks or slowly moving charged particle signature. Such exotic charged track signatures are intensively looked for by ATLAS [84, 85] and CMS $[86,87]$ and also can be investigated by the MoEDAL experiment [88]. We calculate the projected limits obtained from anomalous charged track searches for various simplified models and discuss an interplay with the dark matter relic abundance obtained by the co-annihilation mechanism in the next section. 

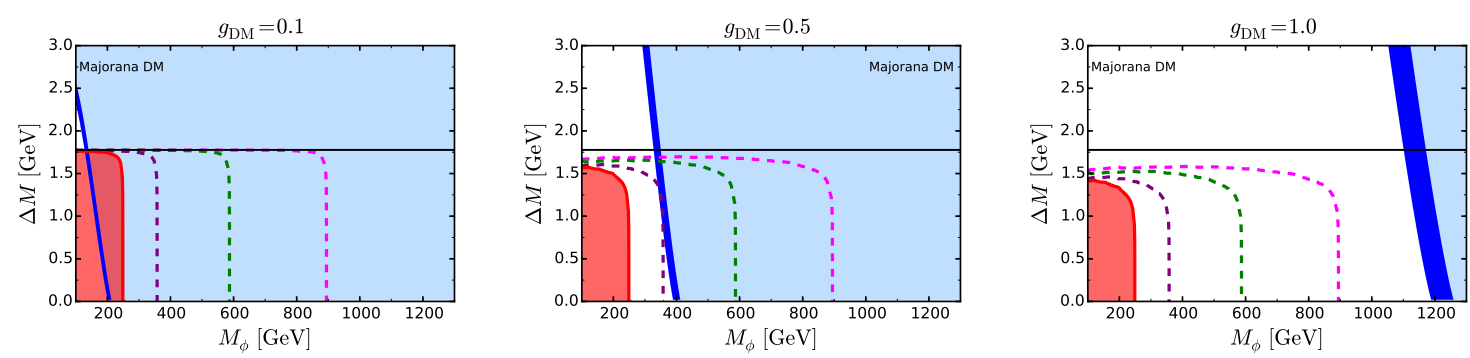

Figure 6. The co-annihilation strip and collider searches for Majorana DM and a long-lived charged scalar in the Simplified Model 1a. The dark-blue region satisfies the correct dark matter relic abundance within $3 \sigma$, the light-blue region overproduces the dark matter energy density. The horizontal black line indicates the mass of the $\tau$ lepton. The region coloured in red corresponds to current HSCP limits at the LHC for center-of-mass energy of $8 \mathrm{TeV}$ and $18.8 \mathrm{fb}^{-1}$. The three dashed lines (purple, green and magenta) correspond to our projections for center-of-mass energy of $13 \mathrm{TeV}$ and 30,300 and $3000 \mathrm{fb}^{-1}$ of integrated luminosity respectively.

\section{Results}

\subsection{Model 1a: Majorana fermion dark matter}

The first simplified model we consider has a Majorana fermion singlet dark matter, $\chi=\chi$, and a complex scalar co-annihilation partner, $\left(\eta^{+}, \eta^{-}\right)=\left(\phi^{*}, \phi\right)=\left(\phi^{+}, \phi^{-}\right)$. We extend the SM Lagrangian as:

$$
\begin{aligned}
\mathcal{L} & =\mathcal{L}_{\mathrm{SM}}+\mathcal{L}_{\mathrm{DM}}+\mathcal{L}_{\mathrm{CAP}}+\mathcal{L}_{\text {int }}, \\
\mathcal{L}_{\mathrm{DM}} & =\frac{1}{2} \chi\left(i \not \supset-m_{\mathrm{DM}}\right) \chi \\
\mathcal{L}_{\mathrm{CAP}} & =\left|D_{\mu} \phi\right|^{2}-M_{\phi}^{2}|\phi|^{2} \\
\mathcal{L}_{\text {int }} & =g_{\mathrm{DM}} \phi^{*} \chi \tau_{R}+\text { h.c. }
\end{aligned}
$$

where $M_{\phi}=m_{\mathrm{DM}}+\Delta M$ and the covariant derivative $D_{\mu}$ contains the $\mathrm{U}(1)_{\mathrm{Y}}$ gauge field. This simplified model has a particular interest since it can be realised in SUSY models by identifying $\chi$ as the Bino and $\phi$ as the right-handed stau. We, however, stress that the model is also interesting on its own right because it is gauge invariant and renormalizable. The searches at LEP have already excluded charged particles with mass below $\simeq 100 \mathrm{GeV}$ [8991], and we focus on the region with $M_{\phi} \gtrsim 100 \mathrm{GeV}$.

We show our numerical results for the Simplified Model 1a in figure 6. The three plots correspond to different values of the dark matter coupling: $g_{\mathrm{DM}}=0.1,0.5$ and 1.0 from left to right. The dark-blue region satisfies the correct dark matter relic abundance within $3 \sigma$, and the light-blue area to the right of it gives a relic abundance which exceeds the observed value and overcloses the universe. The red region corresponds to the current $95 \%$ CL excluded region obtained by the heavy stable charged particle (HSCP) searches at the LHC using $8 \mathrm{TeV}$ data with $18.8 \mathrm{fb}^{-1}$ integrated luminosity [87]. The contours bounded by the purple, green and magenta dashed lines (from left to right) are projected limits assuming $13 \mathrm{TeV}$ LHC with 30,300 and $3000 \mathrm{fb}^{-1}$ integrated luminosities, respectively. 
These projections are obtained by starting with the analysis conducted by CMS [87] of the $8 \mathrm{TeV}$ data, and interpolating it to higher energies and luminosities following the Collider Reach method [92]. ${ }^{5}$ We validated our computational approach by reproducing the $8 \mathrm{TeV}$ limit on the long-lived stau calculated in [94]. The limit can also be presented as a function of the lifetime and mass of $\phi$. Such limits are given in appendix B.

In figure 6 , the horizontal line represents $\Delta M=m_{\tau}$. One can see, as expected, that the limit from the HSCP searches is absent if $\Delta M>m_{\tau}$ since $\phi^{ \pm}$decays before reaching the tracker. Once $\Delta M$ gets smaller than $m_{\tau}$, the propagation path of the $\phi$ charged scalar $c \tau_{\phi}$ reaches and then exceeds the detector scale, $\mathcal{O}(100) \mathrm{cm}$, although the exact $\Delta M$ needed for exclusion depends also on $g_{\mathrm{DM}}$ since the lifetime is inversely proportional to $g_{\mathrm{DM}}^{2}$. For $g_{\mathrm{DM}}=0.1$, the HSCP searches can have strong sensitivities as far as $\Delta M<m_{\tau}$, whilst $\Delta M \lesssim 1.5 \mathrm{GeV}$ is required for $g_{\mathrm{DM}}=0.5$ and 1 . The model can be constrained at the LHC only when there is a large production cross-section for $p p \rightarrow \phi^{+} \phi^{-}$. The sensitivity of the HSCP search therefore has a strong dependence on $M_{\phi}$. If $\Delta M<1.3 \mathrm{GeV}, M_{\phi}<240 \mathrm{GeV}$ is already ruled out by the current data, and the 95\% CL projected limits are estimated as $M_{\phi}<330,580$ and $870 \mathrm{GeV}$ for $13 \mathrm{TeV}$ LHC with 30,300 and $3000 \mathrm{fb}^{-1}$ integrated luminosities, respectively. These limits are almost independent of $g_{\mathrm{DM}}$ and $\Delta M$ as long as $\Delta M<1.3 \mathrm{GeV}$.

We have also shown the constraints from the DM relic density in the same plots. The dark-blue strip in figure 6 represents the region where the DM relic density, computed by MicrOMEGAs 4.1 .5 [67], is consistent with the latest Planck satellite measurement $\Omega_{\mathrm{DM}} h^{2}=0.1197 \pm 0.0022$ [1] within the $3-\sigma$ level. Note that the DM is overproduced on the right of the dark-blue strip, where this region is shaded with light-blue. Conversely, the DM is underproduced on the left of the dark-blue strip. This region may not be excluded phenomenologically since there may be another component for the DM, whose relic density makes up the remaining part of the $\Omega_{\mathrm{DM}} h^{2}$. We can therefore identify the white region as the currently allowed region by the LHC and the DM relic density constraints.

As we have discussed in section 3 , the relic density depends on $\Delta M$ through the coannihilation mechanism, which can be seen clearly in figure 6 . The mass and the dark sector coupling also affect the value of the relic density. To investigate this behaviour in more detail, in figure 7 we present a scan of the $\left(g_{\mathrm{DM}}, m_{\mathrm{DM}}\right)$ plane in our Simplified Model 1a over the mass splittings in the region $0 \leq \Delta M \leq 1 \mathrm{GeV}$. The dark-blue strip gives the correct relic density within $3 \sigma$. As previously discussed, the dependence on $g_{\mathrm{DM}}$ is weak if $g_{\mathrm{DM}} \ll 1$, since the $\left\langle\sigma_{\text {eff }} v\right\rangle$ is almost entirely determined by the $\phi^{+} \phi^{-} \rightarrow S M$ particles, which is independent of $g_{\mathrm{DM}}$. Once $g_{\mathrm{DM}}$ gets as large as the $\mathrm{U}(1)_{\mathrm{Y}}$ gauge coupling, the second process, $\phi^{ \pm} \chi \rightarrow S M$ particles, becomes important, and the dependence on $g_{\mathrm{DM}}$ enters into $\Omega_{\mathrm{DM}} h^{2}$. For very large $g_{\mathrm{DM}}$, the process $\phi^{+} \phi^{+} \rightarrow \tau^{+} \tau^{+}$(and its conjugate), exchanging $\chi$ in the $t$-channel, becomes dominant since it does not incur the chiral suppression and the cross-section is proportional to $g_{\mathrm{DM}}^{4}$. Because the DM relic density is inversely proportional to $\left\langle\sigma_{\text {eff }} v\right\rangle$, the constraint of the DM overproduction excludes small $g_{\mathrm{DM}}$ regions depending on

\footnotetext{
${ }^{5} \mathrm{~A}$ fast recasting method for a HSCP search has been proposed in [93]. We opt for the Collider Reach method, since our main focus is to extrapolate the existing limit to higher energies and luminosities.
} 


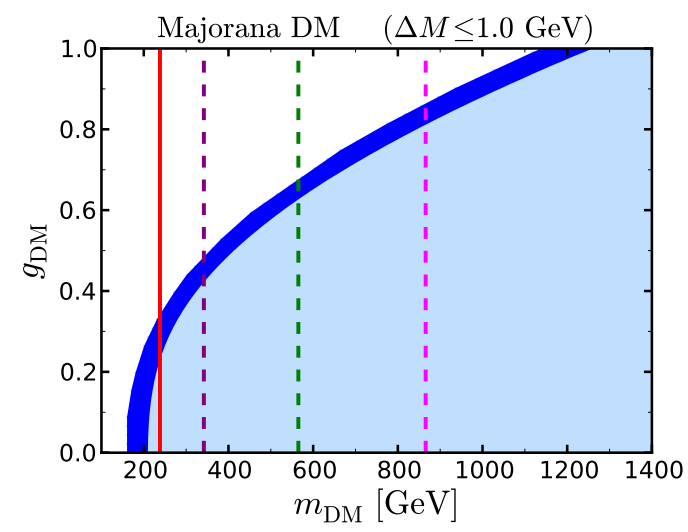

Figure 7. Model 1a: plot of the coupling $g_{\mathrm{DM}}$ versus the dark matter mass $m_{\mathrm{DM}}=m_{\chi}$. We scan over $\Delta M \leq 1 \mathrm{GeV}$, where $\Delta M=M_{\phi}-m_{\chi}$, this is the mass region where the HSCP limits are independent of the coupling $g_{\mathrm{DM}}$. The dark blue band satisfies the correct DM relic abundance within $3 \sigma$, the region in light blue overproduces the amount of DM. The colour-coding for the exclusion regions is the same as in the previous figure.

$m_{\mathrm{DM}}$. From this plot we conclude that the high luminosity LHC at $3000 \mathrm{fb}^{-1}$ can explore almost the entire region with $g_{\mathrm{DM}} \lesssim 1$ except for a small segment around $g_{\mathrm{DM}} \sim 0.9$, $m_{\mathrm{DM}} \sim 1 \mathrm{TeV}$.

\subsection{Model 1b: effect of L-R mixing}

In SUSY models we often encounter the situation where the DM and the lighter stau, $\widetilde{\tau}_{1}$ (co-annihilation partner), interact with both left and right-handed $\tau$-leptons via the L-R mixing in the stau sector. To study this case, we extend the previous simplified model such that the co-annihilation partner $\phi$ can couple to both $\tau_{L}$ and $\tau_{R}$. We will now construct our simplified model by starting initially with the $\mathrm{SU}(2)_{\mathrm{L}} \times \mathrm{U}(1)_{\mathrm{Y}}$ invariant formulation involving a minimal particle content required for the DM fermion, the co-annihilation scalar(s), and the SM leptons. We thus introduce a scalar $\mathrm{SU}(2)$ doublet $\Phi_{L}^{T}=\left(\phi_{\nu}, \phi_{L}\right)$ and a singlet $\phi_{R}$ with the same hyper-charges as those of the SM doublet $l_{3}^{T}=\left(\nu_{\tau}, \tau_{L}\right)$ and the singlet $\tau_{R}$, respectively. We then write down their Yukawa interactions with the DM Majorana fermion $\chi$ as follows,

$$
\sqrt{2} g^{\prime} Y_{l} \Phi_{L}^{\dagger} \chi l_{3}+\sqrt{2} g^{\prime} Y_{e} \phi_{R}^{*} \chi \tau_{R}+\text { h.c. }
$$

where $g^{\prime} \simeq 0.36$ is the $\mathrm{U}(1)_{\mathrm{Y}}$ gauge coupling and $Y_{l}=-\frac{1}{2}$ and $Y_{e}=1$ are the corresponding hyper-charges. These terms are analogous to the bino-stau-tau interaction in SUSY models.

After the electroweak symmetry breaking, the scalars $\phi_{L}$ and $\phi_{R}$ will generically mix with each other forming two mass eigenstates, the lighter of which,

$$
\phi=\cos \theta \phi_{L}+\sin \theta \phi_{R},
$$

we identify as the co-annihilation particle of our simplified model. The mixing angle $\theta$ will be a free parameter in the simplified model. After integrating out the heavier scalar 

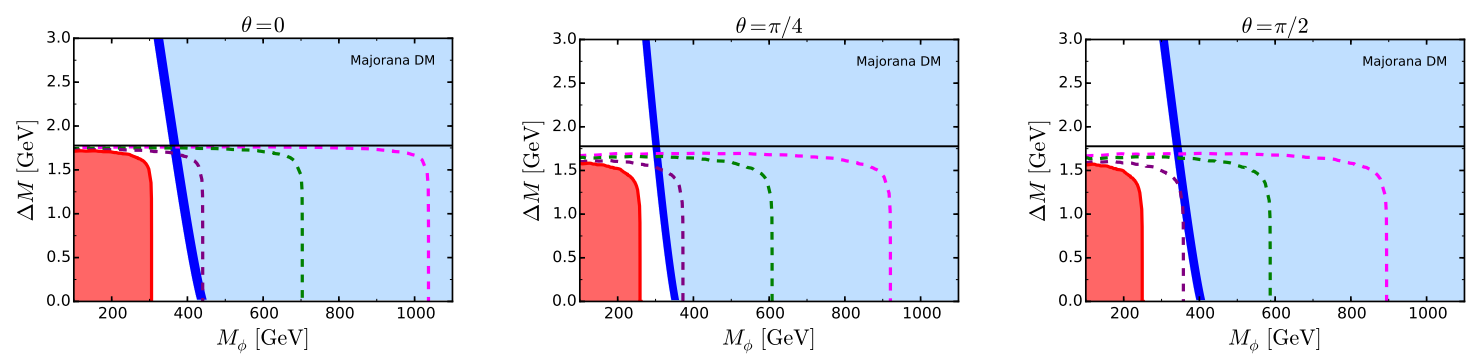

Figure 8. Model 1b: $\phi-\chi$ co-annihilation strip and collider searches. The dark-blue region satisfies the correct dark matter relic abundance within $3 \sigma$, the light-blue region overproduces the dark matter energy density. The horizontal black line corresponds to the mass of the $\tau$ lepton. The region coloured in red corresponds to current HSCP limits for center-of-mass energy of $8 \mathrm{TeV}$ and $18.8 \mathrm{fb}^{-1}$. The three dashed lines (purple, green and magenta) correspond to our projections for center-of-mass energy of $13 \mathrm{TeV}$ and 30,300 and $3000 \mathrm{fb}^{-1}$ of integrated luminosity respectively.

eigenstate, the interaction terms in eq. (5.2) reduce to the simplified model interaction

$$
\mathcal{L}_{\text {int }}=g_{L} \phi^{*} \chi \tau_{L}+g_{R} \phi^{*} \chi \tau_{R}+\text { h.c. },
$$

with the two couplings given by

$$
g_{L}=\frac{1}{\sqrt{2}} g^{\prime} \cos \theta, \quad g_{R}=-\sqrt{2} g^{\prime} \sin \theta .
$$

In the same way, the interaction of $\phi$ with $\gamma, Z$ and $W^{ \pm}$can be obtained by extracting $\phi$ from the kinetic terms $\left|D_{\mu} \Phi_{L}\right|^{2}+\left|D_{\mu} \phi_{R}\right|^{2}$. This defines our Simplified Model 1b, which is determined in terms of three free parameters: $\theta, M_{\phi}$ and $\Delta M=M_{\phi}-m_{\chi}$.

We show in figure 8 the constraints in the $\left(M_{\phi}, \Delta M\right)$ plane for the Simplified Model $1 \mathrm{~b}$ for the following parameter choices: $\theta=0$ for $\phi=\phi_{L}$ (left plot), $\theta=\pi / 4$ for $\phi=$ $\left(\phi_{L}+\phi_{R}\right) / \sqrt{2}$ (central plot) and $\theta=\pi / 2$ for $\phi=\phi_{R}$ (plot on the right). We note that $\theta=\pi / 2$ corresponds to Model-1a with $\left|g_{\mathrm{DM}}\right|=\sqrt{2} g^{\prime} \simeq 0.5$. Therefore the right plot of figure 8 resembles the second plot of figure 6 . One can see that turning on $g_{L}$ makes the LHC constraint tighter. The current HSCP LHC-8 TeV limit on the co-annihilation partner mass increases from $220 \mathrm{GeV}$ to $300 \mathrm{GeV}$ as $\theta$ changes from $\pi / 2$ to 0 . This is because the interaction strength of the $q \bar{q} \rightarrow(\gamma / Z)^{*} \rightarrow \phi^{+} \phi^{-}$process increases due to inclusion of the $\mathrm{SU}(2)_{\mathrm{L}}$ coupling found in $\left|D_{\mu} \Phi_{L}\right|^{2}$.

The dependences of the DM relic density and the lifetime of the co-annihilation partner on $\theta$ are more complicated, and shown in figure 9. Here we plot $\Omega_{\mathrm{DM}} h^{2}$ (solid lines) and $\tau_{\phi}$ (dashed lines) as functions of $\theta$ by fixing $m_{\chi}=300 \mathrm{GeV}$ and varying $\Delta M=1.2,1.4$ and $1.6 \mathrm{GeV}$. We see that $\Omega_{\mathrm{DM}} h^{2}$ is globally minimized at $\theta=0$ and $\pi\left(\phi=\phi_{L}\right)$ due to the relatively large $\mathrm{SU}(2)_{\mathrm{L}}$ coupling. Another local minimum is found at $\theta=\pi / 2\left(\phi=\phi_{R}\right)$. The relic density has two local maxima implying that there is a cancellation in $\left\langle\sigma_{\text {eff }} v\right\rangle$ among $g_{L}$ and $g_{R}$ terms in eq. (5.4). The interference between $g_{L}$ and $g_{R}$ terms can also be observed in the lifetime of $\phi$. Unlike $\Omega_{\mathrm{DM}} h^{2}, \tau_{\phi}$ is minimized (maximized) at $\theta \simeq \frac{3 \pi}{8}\left(\frac{7 \pi}{8}\right)$. 


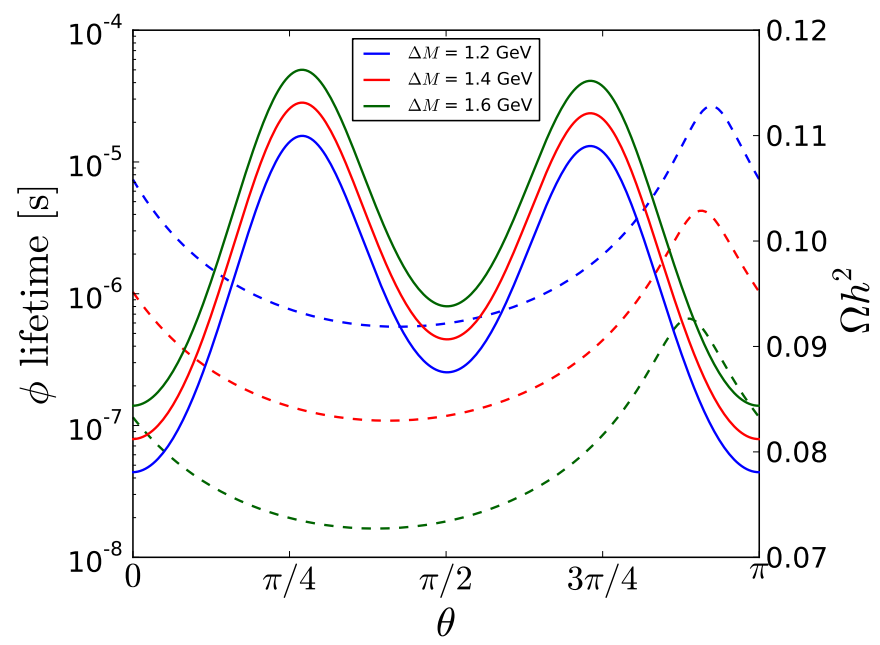

Figure 9. The lifetime of $\phi^{ \pm}$(dashed) and the DM relic density $\Omega h^{2}$ (solid) as functions of the L-R mixing parameter $\theta$. The DM mass is fixed at $300 \mathrm{GeV}$ and $\Delta M$ is varied as 1.2 (blue), 1.4 (red) and 1.6 (green) GeV.

\subsection{Model 2: scalar dark matter}

In this section we consider Simplified Model 2 where the DM particle is a real singlet scalar, $\chi=S$, and the co-annihilation partner is a Dirac fermion, $\left(\eta^{+}, \eta^{-}\right)=(\bar{\Psi}, \Psi)=\left(\Psi^{+}, \Psi^{-}\right)$. We take $\Psi$ to have the same quantum numbers as $\tau_{R}$ except for the $Z_{2}$ (dark sector) charge. The Lagrangian is given as:

$$
\begin{aligned}
\mathcal{L} & =\mathcal{L}_{\mathrm{SM}}+\mathcal{L}_{\mathrm{DM}}+\mathcal{L}_{\mathrm{CAP}}+\mathcal{L}_{\text {int }}, \\
\mathcal{L}_{\mathrm{DM}} & =\frac{1}{2}\left(\partial_{\mu} S\right)^{2}-\frac{1}{2} m_{\mathrm{DM}}^{2} S^{2}, \\
\mathcal{L}_{\mathrm{CAP}} & =\bar{\Psi}\left(i \not D-M_{\Psi}\right) \Psi, \\
\mathcal{L}_{\text {int }} & =g_{\mathrm{DM}} S \bar{\Psi} P_{R} \tau+\text { h.c. },
\end{aligned}
$$

where $M_{\Psi}=m_{\mathrm{DM}}+\Delta M$ and $P_{R}=\frac{1+\gamma_{5}}{2}$ is the right-handed projection operator for Dirac spinors. This simplified model can be realised for example in models with extra dimensions by regarding $\Psi$ as the first excited Kaluza-Klein (KK) mode of the $\tau$ and $S$ as a heavy and stable singlet, such as the first KK-mode of the Higgs boson [95, 96] or a scalar photon in $D \geq 6$ theories [96, 97]. In such models, the approximate mass-degeneracy, or a compressed spectrum between $m_{\chi}$ and $M_{\Psi}$, resulting in $\Delta M \ll m_{\mathrm{DM}}$, which is assumed in this paper, is justified because the mass of each of the KK modes for different particles is dominated by an universal contribution that is inversely proportional to the size of the extra dimension(s). As in the case of Simplified Model 1a, this model is manifestly gauge invariant and renormalizable.

We note that a term $|H|^{2} S^{2}$ is also allowed by the symmetry. After the electroweak symmetry breaking, this term induces a 3 -point interaction $h S S$ that gives the contribution to the direct detection as well as $\Omega_{\mathrm{DM}} h^{2}$. A phenomenological implication of this term has 

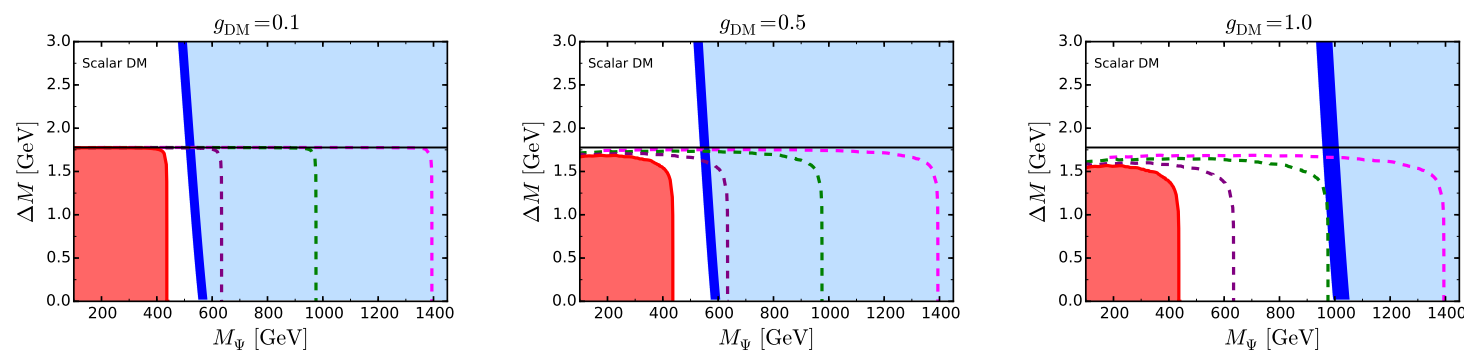

Figure 10. Model 2: the co-annihilation strip and collider searches for scalar DM and a long-lived charged Dirac fermion $\Psi$. The dark-blue region satisfies the correct dark matter relic abundance within $3 \sigma$, the light-blue region overproduces the dark matter energy density. The horizontal black line corresponds to the mass of the $\tau$ lepton. The region coloured in red corresponds to current HSCP limits for center-of-mass energy of $8 \mathrm{TeV}$ and $18.8 \mathrm{fb}^{-1}$. The three dashed lines (purple, green and magenta) correspond to our projections for center-of-mass energy of $13 \mathrm{TeV}$ and 30,300 and $3000 \mathrm{fb}^{-1}$ of integrated luminosity respectively.

been well studied in the literature [58, 98-102]. Since the aim of this paper is to primarily study the effect of co-annihilation, we simply assume that the coefficient of this term is small or otherwise exclude it from our simplified model.

Figure 10 shows our numerical results of this simplified model for $g_{\mathrm{DM}}=0.1,0.5$ and 1.0 from left to right. Comparing it with figure 6, one can see that the LHC limits are tightened but also the preferred co-annihilation partner mass by the relic density gets shifted to higher values. This is because the number of degrees freedom for $\Psi$ is doubled compared to $\phi$. Also, the production cross-section of the co-annihilation partners is enhanced compared to Model-1a because $q \bar{q} \rightarrow \Psi^{+} \Psi^{-}$does not incur velocity suppression near the threshold. The current bound from the HSCP search excludes $M_{\Psi} \lesssim 410 \mathrm{GeV}$ and the projected sensitivity reaches 600,950 and $1350 \mathrm{GeV}$ for the $13 \mathrm{TeV}$ LHC with 30,300 and $3000 \mathrm{fb}^{-1}$ integrated luminosity, respectively. These current and projected limits are independent of $g_{\mathrm{DM}}$ and $\Delta M$ as long as $\Delta M \lesssim 1.5 \mathrm{GeV}$.

The preferred co-annihilation partner mass required by the relic density (the dark-blue strip) is found around $M_{\Psi} \simeq 500-600 \mathrm{GeV}$ for $g_{\mathrm{DM}}=0.1$ and 0.5 , and $M_{\Psi} \simeq 950-1050 \mathrm{GeV}$ for $g_{\mathrm{DM}}=1.0$. The impact of $g_{\mathrm{DM}}$ and $m_{\mathrm{DM}}$ on $\Omega_{\mathrm{DM}} h^{2}$ can be seen more clearly in figure 11, where limits from the LHC and $\Omega_{\mathrm{DM}} h^{2}$ are plotted in the $\left(m_{\mathrm{DM}}, g_{\mathrm{DM}}\right)$ plane scanning $\Delta M$ in the $[0,1.2] \mathrm{GeV}$ range. In this plot, one can see the DM relic density is not sensitive to $g_{\mathrm{DM}}$ until $g_{\mathrm{DM}} \lesssim 0.5$. This is because the $\left\langle\sigma_{\text {eff }} v\right\rangle$ is determined by the process $\Psi^{+} \Psi^{-} \rightarrow S M$ particles, which is independent of $g_{\mathrm{DM}}$. For $g_{\mathrm{DM}}>0.5$, the dependence enters through, i.e., $\Psi^{ \pm} \chi \rightarrow S M$ particles $\left(\left\langle\sigma_{\text {eff }} v\right\rangle \propto g_{\mathrm{DM}}^{2}\right)$ and $\Psi^{ \pm} \Psi^{ \pm} \rightarrow \tau^{ \pm} \tau^{ \pm}$exchanging $S$ in the $t$-channel $\left(\left\langle\sigma_{\mathrm{eff}} v\right\rangle \propto g_{\mathrm{DM}}^{4}\right)$. Considering the limit of the DM overproduction and the HSCP searches, one can see that the entire parameter region with $g_{\mathrm{DM}} \lesssim 1.0$ will be explored by the LHC Run- 2 with $3000 \mathrm{fb}^{-1}$ of integrated luminosity. 


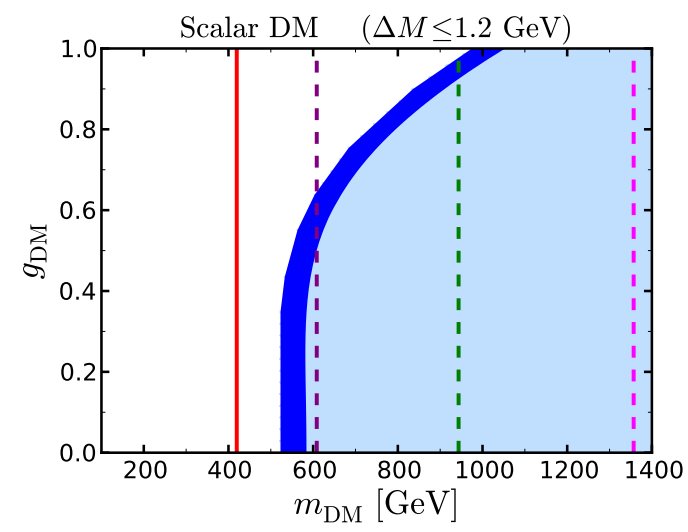

Figure 11. Model 2: plot of the coupling $g_{\mathrm{DM}}$ versus the dark matter mass $m_{\mathrm{DM}}=m_{S}$. We scan over $\Delta M \in[0,1.2 \mathrm{GeV}]$, where $\Delta M=M_{\Psi}-m_{S}$. The dark blue band satisfies the correct DM relic abundance within $3 \sigma$, the region in light blue overproduces the amount of DM. The colour-coding for the exclusion regions is the same as in the previous figure.

\subsection{Model 3: vector dark matter}

We now study the case in which the co-annihilation partner is a Dirac fermion, $\left(\eta^{+}, \eta^{-}\right)=$ $(\bar{\Psi}, \Psi)=\left(\Psi^{+}, \Psi^{-}\right)$, as in Model-2 but the dark matter is a neutral vector boson, $\chi=V_{\mu}$. We modify the Lagrangian eq. (5.6) with

$$
\begin{aligned}
\mathcal{L}_{\mathrm{DM}} & =\frac{1}{4}\left(\partial_{\mu} V_{\nu}-\partial_{\nu} V_{\mu}\right)^{2}+\frac{1}{2} m_{\mathrm{DM}}^{2} V_{\mu} V^{\mu}, \\
\mathcal{L}_{\text {int }} & =g_{\mathrm{DM}} V^{\mu} \bar{\Psi} \gamma_{\mu} P_{R} \tau+\text { h.c. }
\end{aligned}
$$

Similarly to Model-2, this simplified model can be realised in models with extra dimensions by identifying $V_{\mu}$ as the KK photon and $\Psi$ as the KK $\tau$. It may also be possible to interpret $V_{\mu}$ as a $\rho$ meson and $\Psi$ as a baryon in a new strong sector in composite models.

We show our numerical results of this model in figure 12 , where $g_{\mathrm{DM}}=0.1,0.5$ and 0.7 are examined from left to right. One can see that the current and projected LHC limits are almost identical to those found in Model-2, since those models have the same co-annihilation partner $\Psi$, and the relevant production process $q \bar{q} \rightarrow(\gamma / Z)^{*} \rightarrow \Psi \bar{\Psi}$ is independent of the spin of the DM. On the other hand, the relic density constraint is quite different from the corresponding constraint in Model-2. Interestingly, this model has larger $\Omega_{\mathrm{DM}} h^{2}$ for $g_{\mathrm{DM}}=0.1$ compared to Model-2. In the limit $g_{\mathrm{DM}} \ll 1$, eq. (3.2) implies

$$
\frac{\left.\left\langle\sigma_{\text {eff }} v\right\rangle\right|_{\text {Model } 2}}{\left.\left\langle\sigma_{\text {eff }} v\right\rangle\right|_{\text {Model } 3}} \simeq \frac{\left(\mathrm{g}_{V_{\mu}}+\mathrm{g}_{\Psi}\right)^{2}}{\left(\mathrm{~g}_{S}+\mathrm{g}_{\Psi}\right)^{2}}=\frac{49}{25}
$$

On the other hand, for larger $g_{\mathrm{DM}}$ the DM relic rapidly decreases, as can be seen in figure 13 . This is because the contribution of $V_{\mu} V_{\mu} \rightarrow \tau^{+} \tau^{-}$process is not chiral or velocity suppressed in this model and it has a strong dependency on $g_{\mathrm{DM}}:\left\langle\sigma\left(V_{\mu} V_{\mu} \rightarrow \tau^{+} \tau^{-}\right) v\right\rangle \propto g_{\mathrm{DM}}^{4}$. One can see from figure 13 that a large region of the parameter space can be explored by the LHC and relic density constraints. Nevertheless, the region with $m_{\mathrm{DM}} \gtrsim 1.4 \mathrm{TeV}$ and $g_{\mathrm{DM}} \gtrsim 0.7$ 

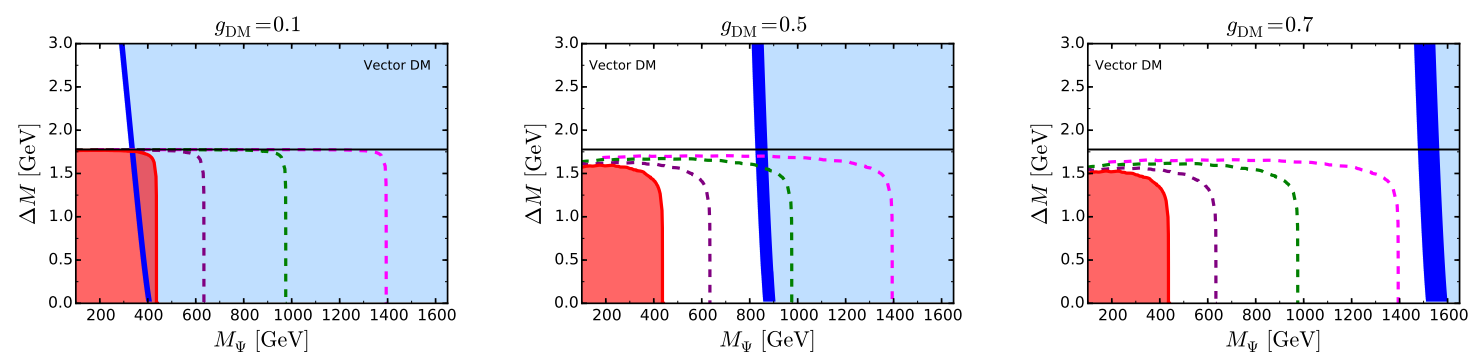

Figure 12. Model 3: the co-annihilation strip and collider searches for vector DM and a long-lived charged Dirac fermion $\Psi$. The dark-blue region satisfies the correct dark matter relic abundance within $3 \sigma$, the light-blue region overproduces the dark matter energy density. The horizontal black line corresponds to the mass of the $\tau$ lepton. The region coloured in red corresponds to current HSCP limits for center-of-mass energy of $8 \mathrm{TeV}$ and $18.8 \mathrm{fb}^{-1}$. The three dashed lines (purple, green and magenta) correspond to our projections for center-of-mass energy of $13 \mathrm{TeV}$ and 30,300 and $3000 \mathrm{fb}^{-1}$ of integrated luminosity respectively.

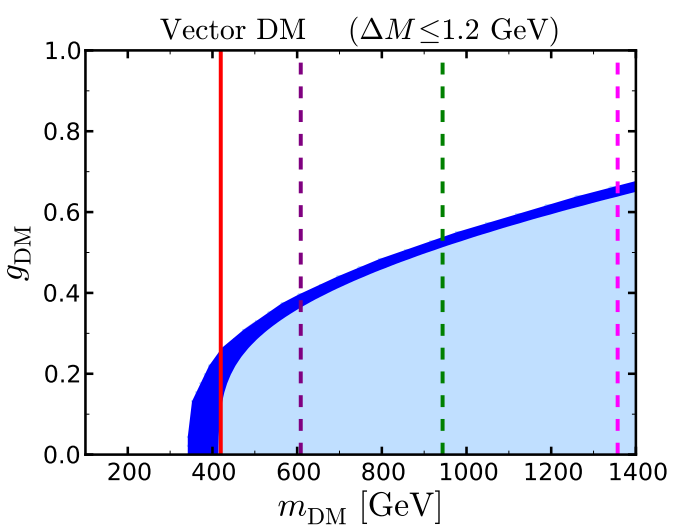

Figure 13. Model 3: plot of the coupling $g_{\mathrm{DM}}$ versus the dark matter mass $m_{\mathrm{DM}}=m_{V}$. We scan over $\Delta M \in[0,1.2 \mathrm{GeV}]$, where $\Delta M=M_{\Psi}-m_{V}$, this is the mass region where the HSCP limits are independent of the coupling $g_{\mathrm{DM}}$. The dark blue band satisfies the correct DM relic abundance within $3 \sigma$, the region in light blue overproduces the amount of DM. The colour-coding for the exclusion regions is the same as in the previous figure.

may be left unconstrained even after the high luminosity LHC with $3000 \mathrm{fb}^{-1}$, although such large values of $g_{\mathrm{DM}}$ might bring sensitivities for the direct and indirect detection experiments, which, however, is beyond the scope of this paper.

\section{Conclusions}

There is a considerable ongoing experimental and theoretical effort dedicated to the discovery of the dark matter. There has been a rapid development in the number and scope of direct and indirect detection experiments, and in LHC and future collider searches of DM. A standard signature to search for dark matter at colliders is the mono-X (or multi-jets) plus missing energy. These searches are being exploited and interpreted in terms of simplified 
dark matter models with mediators. A growing number of the analyses are also dedicated to the direct search of the mediator which can decay back to the SM degrees of freedom.

In this article we considered an alternative DM scenario characterised by simplified models without mediators. Instead they include a co-annihilation partner particle in the dark sector. In the scenarios with a relatively compressed mass spectrum between the DM and its charged co-annihilation partner, the latter plays an important role in lowering the dark matter relic density. The signal we study for collider searches is the pair-production of the co-annihilation partners that then ultimately decay into cosmologically stable dark matter. We have focused on the case when the dark matter candidate and the co-annihilation partner are nearly mass-degenerate, which makes the latter long-lived. Compared to other models of dark matter that rely on signals with missing energy at colliders, in these models the crucial collider signature to look for are tracks of long-lived electrically charged particles.

We have studied for the first time constraints from long-lived particles in the context of simplified dark matter models. We have considered three different scenarios for cosmological DM: a Majorana fermion, a real scalar and a vector dark matter. The model with Majorana DM can be motivated by theories with supersymmetry, such as the binostau co-annihilation strip in the MSSM. The model with vector DM can be motivated by Kaluza-Klein theories of extra dimensions, where the KK photon plays the role of dark matter. Nevertheless, in this work we have advocated for a simple (and arguably more inclusive) purely phenomenological approach and we have considered the couplings and the masses as free parameters.

We have presented a set of simplified models which are complimentary to the standard mediator-based simplified DM models set, and which can be used by the ATLAS and CMS experimental collaborations to interpret their searches for long-lived charged particles to explore this new range of dark matter scenarios which we characterised in terms of 3 to 4 classes of simplified models with as little as 3 free parameters.

\section{Acknowledgments}

This work is supported by STFC through the IPPP consolidated grant. Research of VVK is supported in part by a Royal Society Wolfson Research Merit Award. ADP acknowledges financial support from CONACyT. The work of KS is partially supported by the National Science Centre, Poland, under research grants DEC-2014/15/B/ST2/02157 and DEC-2015/18/M/ST2/00054.

\section{A Indirect detection limits for Model 3}

Unlike Model-1 and Model-2, Model-3 postulates a spin-1 dark matter particle, $V_{\mu}$. The dark matter pair annihilation $V_{\mu} V_{\mu} \rightarrow \tau^{+} \tau^{-}$in the present universe is therefore not chiral suppressed and may be sensitive to indirect detection experiments. We compare the annihilation cross-section computed by micrOMEGAs 4.1 .5 with the upper limit derived from the gamma-ray observations of Milky Way dwarf spheroidal galaxies (dSphs) at the Fermi-LAT satellite [103]. 


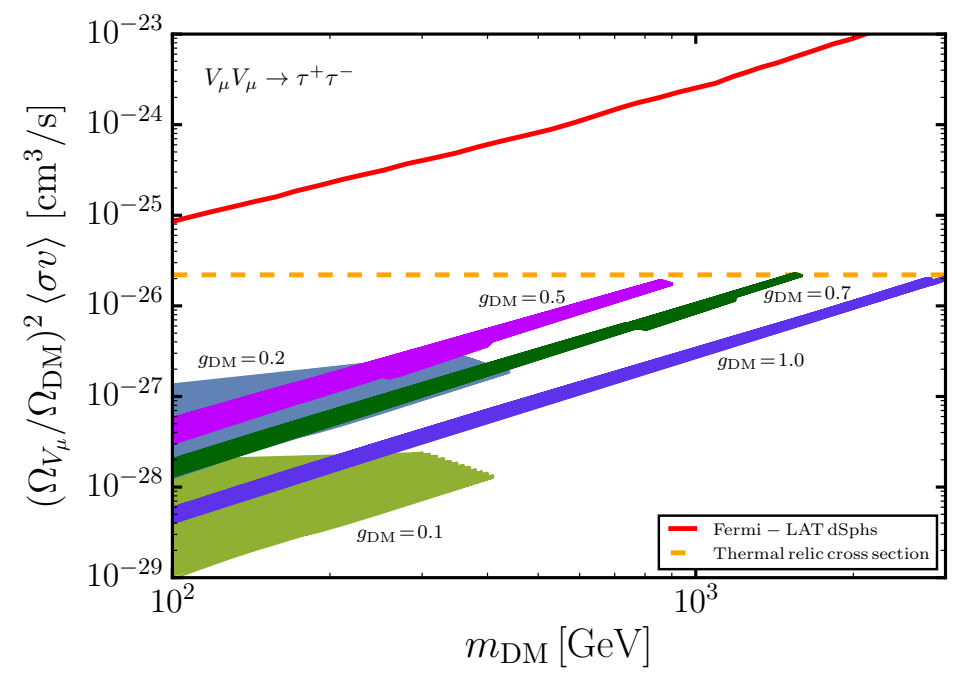

Figure 14. The rate of the dark matter annihilation $V_{\mu} V_{\mu} \rightarrow \tau^{+} \tau^{-}$as a function of the dark matter mass. The red line corresponds to the current limit obtained by the gamma-ray observation of Milky Way dwarf spheroidal galaxies (dSphs) at the Fermi-LAT satellite [103]. The yellow dashed line corresponds to the thermal relic cross-section assuming the pure $V_{\mu} V_{\mu} \rightarrow \tau^{+} \tau^{-}$process. The coloured regions correspond to different values of the coupling $g_{\mathrm{DM}}$ and $\Delta M$ is scanned over the $[0,3] \mathrm{GeV}$ range.

We show our results in figure 14 , where $\Delta M=M_{\Psi}-m_{\mathrm{DM}}$ is scanned over the $[0,3] \mathrm{GeV}$ range and the coloured regions correspond to different values of the coupling $g_{\mathrm{DM}}$, as explained in the figure. In order to confront these with the experimental limit assuming the nominal DM flux, these predictions are rescaled by the square ratio of the calculated relic abundance and the observed one, $\left(\Omega_{V_{\mu}} / \Omega_{\mathrm{DM}}\right)^{2}$ with $\Omega_{\mathrm{DM}} h^{2}=0.1197$. We do not consider points that overproduce the relic abundance, i.e. all the points satisfy $\Omega_{V_{\mu}} h^{2} \leq 0.1197$.

As can be seen, by increasing the dark sector coupling $g_{\mathrm{DM}}$ from 0.5 to 1.0 , the annihilation rate decreases. This is because in this region, the abundance of $V_{\mu}$ is mainly determined by the same annihilation process $V_{\mu} V_{\mu} \rightarrow \tau^{+} \tau^{-}$in the early universe and $\left(\Omega_{V_{\mu}} / \Omega_{\mathrm{DM}}\right)^{2}$ decreases more rapidly than the increase of the present time annihilation crosssection. The situation is different for smaller values of $g_{\mathrm{DM}}$, where $\Omega_{V_{\mu}} h^{2}$ is determined by the co-annihilation mechanism and the annihilation rate of $\Psi^{+} \Psi^{-} \rightarrow S M$ particles, which does not depend on $g_{\mathrm{DM}}$, as discussed in section 3. One can therefore see that going from $g_{\mathrm{DM}}=0.1$ to 0.5 , the annihilation rate increases.

The red line in figure 14 shows the Fermi-LAT limit assuming dark matter annihilation into the $\tau^{+} \tau^{-}$final state. As can be seen, the predicted rate is more than two order of magnitude smaller than the current limit across the parameter region.

\section{B Limits in the mass vs lifetime plane}

The current and projected limits obtained from the heavy stable charged particle searches shown in section 5 can also be presented in a more model-independent fashion by plotting 

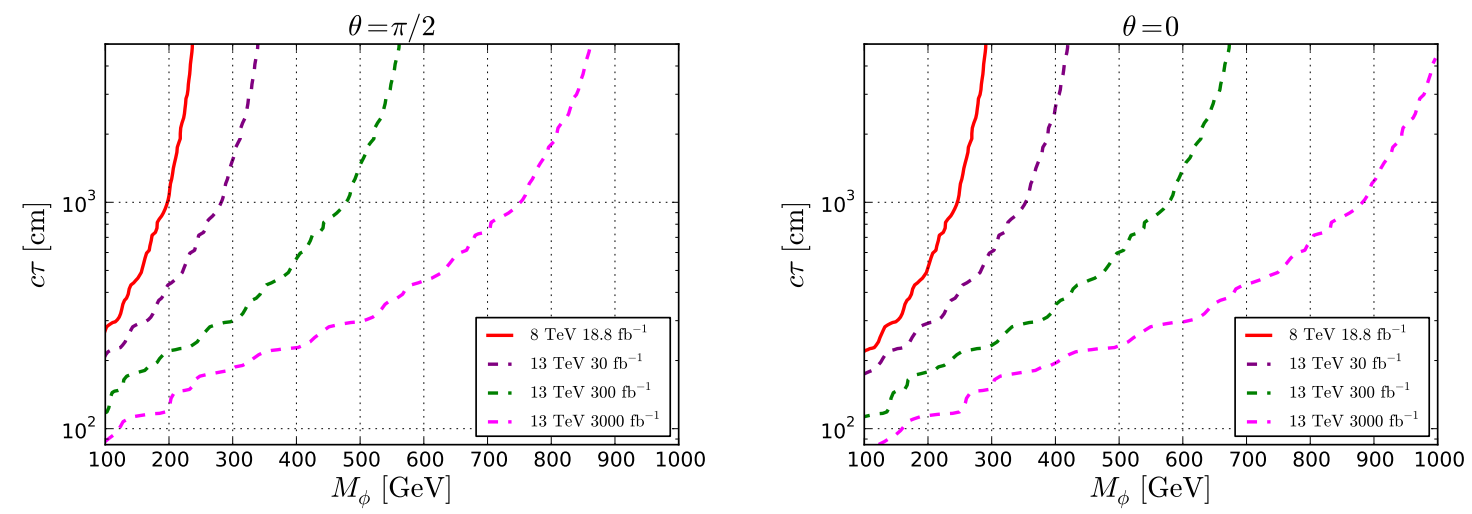

Figure 15. The $8 \mathrm{TeV}$ (solid) and projected $13 \mathrm{TeV}$ (dashed) limits from HSCP searches at the LHC for pair-production of the scalar co-annihilation partner, $\phi^{ \pm}$. The projected limits correspond to the $13 \mathrm{TeV}$ LHC with 30,300 and $3000 \mathrm{fb}^{-1}$ integrated luminosities.

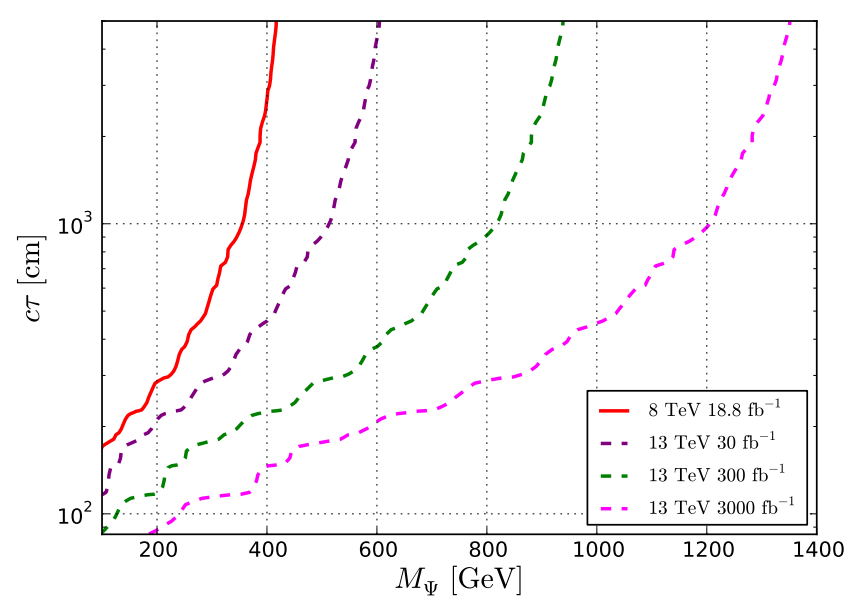

Figure 16. The $8 \mathrm{TeV}$ (solid) and projected $13 \mathrm{TeV}$ (dashed) limits from HSCP searches at the LHC for pair-production of the fermionic co-annihilation partner, $\Psi^{ \pm}$. The projected limits correspond to the $13 \mathrm{TeV}$ LHC with 30,300 and $3000 \mathrm{fb}^{-1}$ integrated luminosities.

on the mass vs lifetime plane. The plots in figure 15 shows the $8 \mathrm{TeV}$ (solid) and projected (dashed) limits for the pair-production of long-lived complex scalar field, $\phi$, as a function of the mass, $M_{\phi}$, and the lifetime times the speed of light, $c \tau$. The left plot assumes $\phi$ has the same quantum number as the right-handed $\tau$ corresponding to Simplified Model 1a. In the right plot, on the other hand, the interaction of $\phi$ is obtained by the procedure explained in section 5.2 (Simplified Model $1 \mathrm{~b}$ ) and taking $\theta=0$. The co-annihilation partner $\phi$ in this case corresponds to the purely left-handed stau in SUSY theories. Figure 16 shows the same limits for the fermionic co-annihilation partner, $\Psi$. These limits are applicable for both Simplified Model 2 and 3 discussed in this paper. 
Open Access. This article is distributed under the terms of the Creative Commons Attribution License (CC-BY 4.0), which permits any use, distribution and reproduction in any medium, provided the original author(s) and source are credited.

\section{References}

[1] Planck collaboration, P.A.R. Ade et al., Planck 2015 results XIII. Cosmological parameters, Astron. Astrophys. 594 (2016) A13 [arXiv:1502.01589] [INSPIRE].

[2] G. Bertone, D. Hooper and J. Silk, Particle dark matter: evidence, candidates and constraints, Phys. Rept. 405 (2005) 279 [hep-ph/0404175] [INSPIRE].

[3] C. Cheung, L.J. Hall, D. Pinner and J.T. Ruderman, Prospects and blind spots for neutralino dark matter, JHEP 05 (2013) 100 [arXiv:1211.4873] [INSPIRE].

[4] H. Baer, V. Barger and D. Mickelson, Direct and indirect detection of higgsino-like WIMPs: concluding the story of electroweak naturalness, Phys. Lett. B 726 (2013) 330 [arXiv:1303.3816] [INSPIRE].

[5] D. Barducci, A. Belyaev, A.K.M. Bharucha, W. Porod and V. Sanz, Uncovering natural supersymmetry via the interplay between the LHC and direct dark matter detection, JHEP 07 (2015) 066 [arXiv: 1504.02472] [INSPIRE].

[6] M. Badziak, A. Delgado, M. Olechowski, S. Pokorski and K. Sakurai, Detecting underabundant neutralinos, JHEP 11 (2015) 053 [arXiv:1506.07177] [INSPIRE].

[7] P. Huang, R.A. Roglans, D.D. Spiegel, Y. Sun and C.E.M. Wagner, Constraints on supersymmetric dark matter for heavy scalar superpartners, Phys. Rev. D 95 (2017) 095021 [arXiv: 1701.02737] [INSPIRE].

[8] M. Badziak, M. Olechowski and P. Szczerbiak, Is well-tempered neutralino in MSSM still alive after 2016 LUX results?, Phys. Lett. B 770 (2017) 226 [arXiv:1701. 05869] [InSPIRE].

[9] E.A. Bagnaschi et al., Supersymmetric dark matter after LHC run 1, Eur. Phys. J. C 75 (2015) 500 [arXiv: 1508.01173] [InSPIRE].

[10] K.J. de Vries et al., The pMSSM10 after LHC run 1, Eur. Phys. J. C 75 (2015) 422 [arXiv: 1504.03260] [INSPIRE].

[11] E. Bagnaschi et al., Likelihood analysis of supersymmetric SU(5) GUTs, Eur. Phys. J. C 77 (2017) 104 [arXiv: 1610.10084] [INSPIRE].

[12] T. Cohen, M. Lisanti, A. Pierce and T.R. Slatyer, Wino dark matter under siege, JCAP 10 (2013) 061 [arXiv: 1307.4082] [InSPIRE].

[13] J. Fan and M. Reece, In wino veritas? Indirect searches shed light on neutralino dark matter, JHEP 10 (2013) 124 [arXiv: 1307.4400] [INSPIRE].

[14] M. Beneke, A. Bharucha, A. Hryczuk, S. Recksiegel and P. Ruiz-Femenia, The last refuge of mixed wino-Higgsino dark matter, JHEP 01 (2017) 002 [arXiv:1611.00804] [INSPIRE].

[15] T. Cohen, J. Kearney, A. Pierce and D. Tucker-Smith, Singlet-doublet dark matter, Phys. Rev. D 85 (2012) 075003 [arXiv:1109.2604] [INSPIRE].

[16] M. Chala, F. Kahlhoefer, M. McCullough, G. Nardini and K. Schmidt-Hoberg, Constraining dark sectors with monojets and dijets, JHEP 07 (2015) 089 [arXiv:1503.05916] [INSPIRE].

[17] M. Beltrán, D. Hooper, E.W. Kolb and Z.C. Krusberg, Deducing the nature of dark matter from direct and indirect detection experiments in the absence of collider signatures of new physics, Phys. Rev. D 80 (2009) 043509 [arXiv:0808.3384] [INSPIRE]. 
[18] Q.-H. Cao, C.-R. Chen, C.S. Li and H. Zhang, Effective dark matter model: relic density, CDMS II, Fermi LAT and LHC, JHEP 08 (2011) 018 [arXiv:0912.4511] [INSPIRE].

[19] J. Goodman, M. Ibe, A. Rajaraman, W. Shepherd, T.M.P. Tait and H.-B. Yu, Constraints on light Majorana dark matter from colliders, Phys. Lett. B 695 (2011) 185 [arXiv:1005.1286] [INSPIRE].

[20] Y. Bai, P.J. Fox and R. Harnik, The Tevatron at the frontier of dark matter direct detection, JHEP 12 (2010) 048 [arXiv: 1005.3797] [INSPIRE].

[21] J. Fan, M. Reece and L.-T. Wang, Non-relativistic effective theory of dark matter direct detection, JCAP 11 (2010) 042 [arXiv: 1008.1591] [INSPIRE].

[22] J. Goodman, M. Ibe, A. Rajaraman, W. Shepherd, T.M.P. Tait and H.-B. Yu, Constraints on dark matter from colliders, Phys. Rev. D 82 (2010) 116010 [arXiv:1008.1783] [INSPIRE].

[23] J.-M. Zheng, Z.-H. Yu, J.-W. Shao, X.-J. Bi, Z. Li and H.-H. Zhang, Constraining the interaction strength between dark matter and visible matter: I. Fermionic dark matter, Nucl. Phys. B 854 (2012) 350 [arXiv:1012.2022] [INSPIRE].

[24] M.R. Buckley, Asymmetric dark matter and effective operators, Phys. Rev. D 84 (2011) 043510 [arXiv: 1104.1429] [INSPIRE].

[25] Z.-H. Yu, J.-M. Zheng, X.-J. Bi, Z. Li, D.-X. Yao and H.-H. Zhang, Constraining the interaction strength between dark matter and visible matter: II. Scalar, vector and spin-3/2 dark matter, Nucl. Phys. B 860 (2012) 115 [arXiv:1112.6052] [INSPIRE].

[26] A. Rajaraman, W. Shepherd, T.M.P. Tait and A.M. Wijangco, LHC bounds on interactions of dark matter, Phys. Rev. D 84 (2011) 095013 [arXiv:1108.1196] [INSPIRE].

[27] K. Cheung, P.-Y. Tseng, Y.-L.S. Tsai and T.-C. Yuan, Global constraints on effective dark matter interactions: relic density, direct detection, indirect detection and collider, JCAP 05 (2012) 001 [arXiv: 1201.3402] [INSPIRE].

[28] R.C. Cotta, J.L. Hewett, M.P. Le and T.G. Rizzo, Bounds on dark matter interactions with electroweak gauge bosons, Phys. Rev. D 88 (2013) 116009 [arXiv:1210.0525] [INSPIRE].

[29] H. Dreiner, D. Schmeier and J. Tattersall, Contact interactions probe effective dark matter models at the LHC, Europhys. Lett. 102 (2013) 51001 [arXiv:1303.3348] [INSPIRE].

[30] U. Haisch, A. Hibbs and E. Re, Determining the structure of dark-matter couplings at the LHC, Phys. Rev. D 89 (2014) 034009 [arXiv:1311.7131] [InSPIRE].

[31] G. Busoni, A. De Simone, E. Morgante and A. Riotto, On the validity of the effective field theory for dark matter searches at the LHC, Phys. Lett. B 728 (2014) 412 [arXiv: 1307.2253] [INSPIRE].

[32] O. Buchmueller, M.J. Dolan and C. McCabe, Beyond effective field theory for dark matter searches at the LHC, JHEP 01 (2014) 025 [arXiv: 1308.6799] [INSPIRE].

[33] G. Busoni, A. De Simone, J. Gramling, E. Morgante and A. Riotto, On the validity of the effective field theory for dark matter searches at the LHC, part II: complete analysis for the s-channel, JCAP 06 (2014) 060 [arXiv:1402.1275] [INSPIRE].

[34] M.R. Buckley, D. Feld and D. Goncalves, Scalar simplified models for dark matter, Phys. Rev. D 91 (2015) 015017 [arXiv: 1410.6497] [InSPIRE].

[35] P. Harris, V.V. Khoze, M. Spannowsky and C. Williams, Constraining dark sectors at colliders: beyond the effective theory approach, Phys. Rev. D 91 (2015) 055009 [arXiv: 1411.0535] [INSPIRE]. 
[36] U. Haisch and E. Re, Simplified dark matter top-quark interactions at the LHC, JHEP 06 (2015) 078 [arXiv: 1503.00691] [INSPIRE].

[37] O. Buchmueller, M.J. Dolan, S.A. Malik and C. McCabe, Characterising dark matter searches at colliders and direct detection experiments: vector mediators, JHEP 01 (2015) 037 [arXiv: 1407.8257] [INSPIRE].

[38] D. Abercrombie et al., Dark matter benchmark models for early LHC run-2 searches: report of the ATLAS/CMS dark matter forum, arXiv:1507.00966 [INSPIRE].

[39] G. Busoni et al., Recommendations on presenting LHC searches for missing transverse energy signals using simplified s-channel models of dark matter, arXiv:1603.04156 [INSPIRE].

[40] T. Golling et al., Physics at a $100 \mathrm{TeV}$ pp collider: beyond the Standard Model phenomena, submitted to Phys. Rept. (2016) [arXiv:1606.00947] [inSPIRE].

[41] P. Harris, V.V. Khoze, M. Spannowsky and C. Williams, Closing up on dark sectors at colliders: from 14 to $100 \mathrm{TeV}$, Phys. Rev. D 93 (2016) 054030 [arXiv:1509.02904] [INSPIRE].

[42] V.V. Khoze, G. Ro and M. Spannowsky, Spectroscopy of scalar mediators to dark matter at the LHC and at $100 \mathrm{TeV}$, Phys. Rev. D 92 (2015) 075006 [arXiv: 1505.03019] [InSPIRE].

[43] A. Berlin, S. Gori, T. Lin and L.-T. Wang, Pseudoscalar portal dark matter, Phys. Rev. D 92 (2015) 015005 [arXiv: 1502.06000] [InSPIRE].

[44] S. Baek, P. Ko, M. Park, W.-I. Park and C. Yu, Beyond the dark matter effective field theory and a simplified model approach at colliders, Phys. Lett. B 756 (2016) 289 [arXiv: 1506. 06556] [INSPIRE].

[45] F. Kahlhoefer, K. Schmidt-Hoberg, T. Schwetz and S. Vogl, Implications of unitarity and gauge invariance for simplified dark matter models, JHEP 02 (2016) 016 [arXiv: 1510.02110] [INSPIRE].

[46] C. Englert, M. McCullough and M. Spannowsky, S-channel dark matter simplified models and unitarity, Phys. Dark Univ. 14 (2016) 48 [arXiv:1604.07975] [INSPIRE].

[47] D. Goncalves, P.A.N. Machado and J.M. No, Simplified models for dark matter face their consistent completions, Phys. Rev. D 95 (2017) 055027 [arXiv:1611.04593] [INSPIRE].

[48] S. Baek, P. Ko and J. Li, Minimal renormalizable simplified dark matter model with a pseudoscalar mediator, Phys. Rev. D 95 (2017) 075011 [arXiv: 1701.04131] [INSPIRE].

[49] M. Bauer, U. Haisch and F. Kahlhoefer, Simplified dark matter models with two Higgs doublets: I. Pseudoscalar mediators, JHEP 05 (2017) 138 [arXiv: 1701.07427] [INSPIRE].

[50] M.J. Baker et al., The coannihilation codex, JHEP 12 (2015) 120 [arXiv:1510.03434] [INSPIRE].

[51] M. Garny, A. Ibarra, M. Pato and S. Vogl, Closing in on mass-degenerate dark matter scenarios with antiprotons and direct detection, JCAP 11 (2012) 017 [arXiv:1207.1431] [INSPIRE].

[52] C. Kelso, J. Kumar, P. Sandick and P. Stengel, Charged mediators in dark matter scattering with nuclei and the strangeness content of nucleons, Phys. Rev. D 91 (2015) 055028 [arXiv: 1411.2634] [INSPIRE].

[53] A. Ibarra, A. Pierce, N.R. Shah and S. Vogl, Anatomy of coannihilation with a scalar top partner, Phys. Rev. D 91 (2015) 095018 [arXiv:1501.03164] [INSPIRE]. 
[54] J. Ellis, F. Luo and K.A. Olive, Gluino coannihilation revisited, JHEP 09 (2015) 127 [arXiv: 1503.07142] [INSPIRE].

[55] M. Buschmann et al., Hunting for dark matter coannihilation by mixing dijet resonances and missing transverse energy, JHEP 09 (2016) 033 [arXiv: 1605.08056] [INSPIRE].

[56] A. Albert et al., Towards the next generation of simplified dark matter models, Phys. Dark Univ. 16 (2017) 49 [arXiv:1607.06680] [INSPIRE].

[57] M. Cirelli, N. Fornengo and A. Strumia, Minimal dark matter, Nucl. Phys. B 753 (2006) 178 [hep-ph/0512090] [INSPIRE].

[58] V.V. Khoze, C. McCabe and G. Ro, Higgs vacuum stability from the dark matter portal, JHEP 08 (2014) 026 [arXiv: 1403.4953] [INSPIRE].

[59] J.R. Ellis, T. Falk and K.A. Olive, Neutralino-stau coannihilation and the cosmological upper limit on the mass of the lightest supersymmetric particle, Phys. Lett. B 444 (1998) 367 [hep-ph/9810360] [INSPIRE].

[60] T. Jittoh, J. Sato, T. Shimomura and M. Yamanaka, Long life stau in the minimal supersymmetric Standard Model, Phys. Rev. D 73 (2006) 055009 [Erratum ibid. D 87 (2013) 019901] [hep-ph/0512197] [INSPIRE].

[61] S. Kaneko, J. Sato, T. Shimomura, O. Vives and M. Yamanaka, Measuring lepton flavor violation at LHC with a long-lived slepton in the coannihilation region, Phys. Rev. D 78 (2008) 116013 [Erratum ibid. D 87 (2013) 039904] [arXiv:0811.0703] [INSPIRE].

[62] T. Jittoh, K. Kohri, M. Koike, J. Sato, T. Shimomura and M. Yamanaka, Stau relic density at the big-bang nucleosynthesis era consistent with the abundance of the light element nuclei in the coannihilation scenario, Phys. Rev. D 82 (2010) 115030 [arXiv:1001.1217] [INSPIRE].

[63] M. Citron, J. Ellis, F. Luo, J. Marrouche, K.A. Olive and K.J. de Vries, End of the CMSSM coannihilation strip is nigh, Phys. Rev. D 87 (2013) 036012 [arXiv:1212.2886] [INSPIRE].

[64] Y. Konishi, S. Ohta, J. Sato, T. Shimomura, K. Sugai and M. Yamanaka, First evidence of the constrained minimal supersymmetric Standard Model is appearing soon, Phys. Rev. D 89 (2014) 075006 [arXiv: 1309. 2067] [INSPIRE].

[65] N. Desai, J. Ellis, F. Luo and J. Marrouche, Closing in on the tip of the CMSSM stau coannihilation strip, Phys. Rev. D 90 (2014) 055031 [arXiv: 1404.5061] [INSPIRE].

[66] K. Griest and D. Seckel, Three exceptions in the calculation of relic abundances, Phys. Rev. D 43 (1991) 3191 [INSPIRE].

[67] G. Bélanger, F. Boudjema, A. Pukhov and A. Semenov, MicrOMEGAs: a program for calculating the relic density in the MSSM, Comput. Phys. Commun. 149 (2002) 103 [hep-ph/0112278] [INSPIRE].

[68] A. Alloul, N.D. Christensen, C. Degrande, C. Duhr and B. Fuks, FeynRules $2.0-a$ complete toolbox for tree-level phenomenology, Comput. Phys. Commun. 185 (2014) 2250 [arXiv: 1310.1921] [INSPIRE].

[69] A. Semenov, LanHEP - a package for automatic generation of Feynman rules from the Lagrangian. Version 3.2, Comput. Phys. Commun. 201 (2016) 167 [arXiv:1412.5016] [INSPIRE].

[70] J. Kopp, L. Michaels and J. Smirnov, Loopy constraints on leptophilic dark matter and internal bremsstrahlung, JCAP 04 (2014) 022 [arXiv:1401.6457] [INSPIRE]. 
[71] LUX collaboration, D.S. Akerib et al., First results from the LUX dark matter experiment at the Sanford Underground Research Facility, Phys. Rev. Lett. 112 (2014) 091303 [arXiv:1310.8214] [INSPIRE].

[72] D.C. Malling et al., After LUX: the LZ program, arXiv:1110.0103 [INSPIRE].

[73] T. Toma, Internal bremsstrahlung signature of real scalar dark matter and consistency with thermal relic density, Phys. Rev. Lett. 111 (2013) 091301 [arXiv:1307.6181] [INSPIRE].

[74] F. Giacchino, L. Lopez-Honorez and M.H.G. Tytgat, Scalar dark matter models with significant internal bremsstrahlung, JCAP 10 (2013) 025 [arXiv:1307.6480] [INSPIRE].

[75] J. Kumar, P. Sandick, F. Teng and T. Yamamoto, Gamma-ray signals from dark matter annihilation via charged mediators, Phys. Rev. D 94 (2016) 015022 [arXiv:1605.03224] [INSPIRE].

[76] FERMI-LAT collaboration, M. Ackermann et al., Search for gamma-ray spectral lines with the Fermi Large Area Telescope and dark matter implications, Phys. Rev. D 88 (2013) 082002 [arXiv: 1305.5597] [INSPIRE].

[77] H.E.S.S. collaboration, A. Abramowski et al., Search for photon-linelike signatures from dark matter annihilations with H.E.S.S., Phys. Rev. Lett. 110 (2013) 041301 [arXiv: 1301.1173] [INSPIRE].

[78] CTA Consortium collaboration, M. Actis et al., Design concepts for the Cherenkov Telescope Array CTA: an advanced facility for ground-based high-energy gamma-ray astronomy, Exper. Astron. 32 (2011) 193 [arXiv: 1008.3703] [INSPIRE].

[79] M. Garny, A. Ibarra, M. Pato and S. Vogl, Internal bremsstrahlung signatures in light of direct dark matter searches, JCAP 12 (2013) 046 [arXiv:1306.6342] [INSPIRE].

[80] J. Alwall et al., The automated computation of tree-level and next-to-leading order differential cross sections and their matching to parton shower simulations, JHEP 07 (2014) 079 [arXiv: 1405.0301] [INSPIRE].

[81] Z.-H. Yu, X.-J. Bi, Q.-S. Yan and P.-F. Yin, Tau portal dark matter models at the LHC, Phys. Rev. D 91 (2015) 035008 [arXiv:1410.3347] [InSPIRE].

[82] M. Low and L.-T. Wang, Neutralino dark matter at 14 TeV and 100 TeV, JHEP 08 (2014) 161 [arXiv: 1404.0682] [INSPIRE].

[83] A. Belyaev, N.D. Christensen and A. Pukhov, CalcHEP 3.4 for collider physics within and beyond the Standard Model, Comput. Phys. Commun. 184 (2013) 1729 [arXiv:1207.6082] [INSPIRE].

[84] ATLAS collaboration, Search for metastable heavy charged particles with large ionization energy loss in pp collisions at $\sqrt{s}=13$ TeV using the ATLAS experiment, Phys. Rev. D 93 (2016) 112015 [arXiv:1604.04520] [INSPIRE].

[85] ATLAS collaboration, Search for heavy long-lived charged R-hadrons with the ATLAS detector in $3.2 \mathrm{fb}^{-1}$ of proton-proton collision data at $\sqrt{s}=13 \mathrm{TeV}$, Phys. Lett. $\mathbf{B} 760$ (2016) 647 [arXiv:1606.05129] [INSPIRE].

[86] CMS collaboration, Search for long-lived charged particles in proton-proton collisions at $\sqrt{s}=13$ TeV, Phys. Rev. D 94 (2016) 112004 [arXiv:1609.08382] [InSPIRE].

[87] CMS collaboration, Constraints on the pMSSM, AMSB model and on other models from the search for long-lived charged particles in proton-proton collisions at $\sqrt{s}=8$ TeV, Eur. Phys. J. C 75 (2015) 325 [arXiv:1502.02522] [InSPIRE]. 
[88] MoEDAL collaboration, B. Acharya et al., The physics programme of the MoEDAL experiment at the LHC, Int. J. Mod. Phys. A 29 (2014) 1430050 [arXiv:1405.7662] [INSPIRE].

[89] ALEPH collaboration, A. Heister et al., Absolute lower limits on the masses of selectrons and sneutrinos in the MSSM, Phys. Lett. B 544 (2002) 73 [hep-ex/0207056] [INSPIRE].

[90] L3 collaboration, P. Achard et al., Search for scalar leptons and scalar quarks at LEP, Phys. Lett. B 580 (2004) 37 [hep-ex/0310007] [INSPIRE].

[91] DELPHI collaboration, J. Abdallah et al., Searches for supersymmetric particles in $e^{+} e^{-}$ collisions up to $208 \mathrm{GeV}$ and interpretation of the results within the MSSM, Eur. Phys. J. C 31 (2003) 421 [hep-ex/0311019] [InSPIRE].

[92] G. Salam and A. Weiler, Collider Reach tool webpage, http://collider-reach.web.cern.ch/.

[93] J. Heisig, A. Lessa and L. Quertenmont, Simplified models for exotic BSM searches, JHEP 12 (2015) 087 [arXiv: 1509.00473] [inSPIRE].

[94] J.A. Evans and J. Shelton, Long-lived staus and displaced leptons at the LHC, JHEP 04 (2016) 056 [arXiv: 1601.01326] [INSPIRE].

[95] T. Flacke, A. Menon and D.J. Phalen, Non-minimal universal extra dimensions, Phys. Rev. D 79 (2009) 056009 [arXiv:0811.1598] [INSPIRE].

[96] S. Arrenberg, L. Baudis, K. Kong, K.T. Matchev and J. Yoo, Kaluza-Klein dark matter: direct detection vis-à-vis LHC, Phys. Rev. D 78 (2008) 056002 [arXiv:0805.4210] [INSPIRE].

[97] B.A. Dobrescu, D. Hooper, K. Kong and R. Mahbubani, Spinless photon dark matter from two universal extra dimensions, JCAP 10 (2007) 012 [arXiv:0706.3409] [INSPIRE].

[98] A. Djouadi, O. Lebedev, Y. Mambrini and J. Quevillon, Implications of LHC searches for Higgs-portal dark matter, Phys. Lett. B 709 (2012) 65 [arXiv:1112.3299] [INSPIRE].

[99] L. Lopez-Honorez, T. Schwetz and J. Zupan, Higgs portal, fermionic dark matter and a Standard Model like Higgs at 125 GeV, Phys. Lett. B 716 (2012) 179 [arXiv:1203.2064] [INSPIRE].

[100] A. Djouadi, A. Falkowski, Y. Mambrini and J. Quevillon, Direct detection of Higgs-portal dark matter at the LHC, Eur. Phys. J. C 73 (2013) 2455 [arXiv:1205.3169] [INSPIRE].

[101] A. Freitas, S. Westhoff and J. Zupan, Integrating in the Higgs portal to fermion dark matter, JHEP 09 (2015) 015 [arXiv: 1506.04149] [INSPIRE].

[102] J. Brooke, M.R. Buckley, P. Dunne, B. Penning, J. Tamanas and M. Zgubic, Vector boson fusion searches for dark matter at the LHC, Phys. Rev. D 93 (2016) 113013 [arXiv: 1603. 07739] [INSPIRE].

[103] Fermi-LAT collaboration, M. Ackermann et al., Searching for dark matter annihilation from milky way dwarf spheroidal galaxies with six years of Fermi Large Area Telescope data, Phys. Rev. Lett. 115 (2015) 231301 [arXiv:1503.02641] [INSPIRE]. 